DEPARTMENT OF THE INTERIOR

UNITED STATES GEOLOGICAL SURVEY

GEORGE OTIS SMITH, DIRECTOR

Water-Supply Paper 345-H

\title{
GROUND-WATER RESOURCES OF THE NILES CONE AND ADJACENT AREAS, CALIFORNIA
}

BY

W. O. CLARK

Prepared in cooperation with the

Department of Engineering of the State of California

Oontributions to the Hydrology of the United States, 1914-H

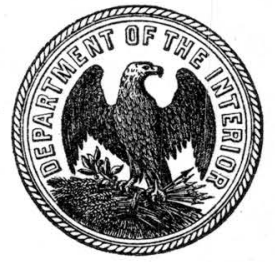

W A SHINGTON

GOVERNMENT PRINTING OFFICE 


\section{CONTENTS.}

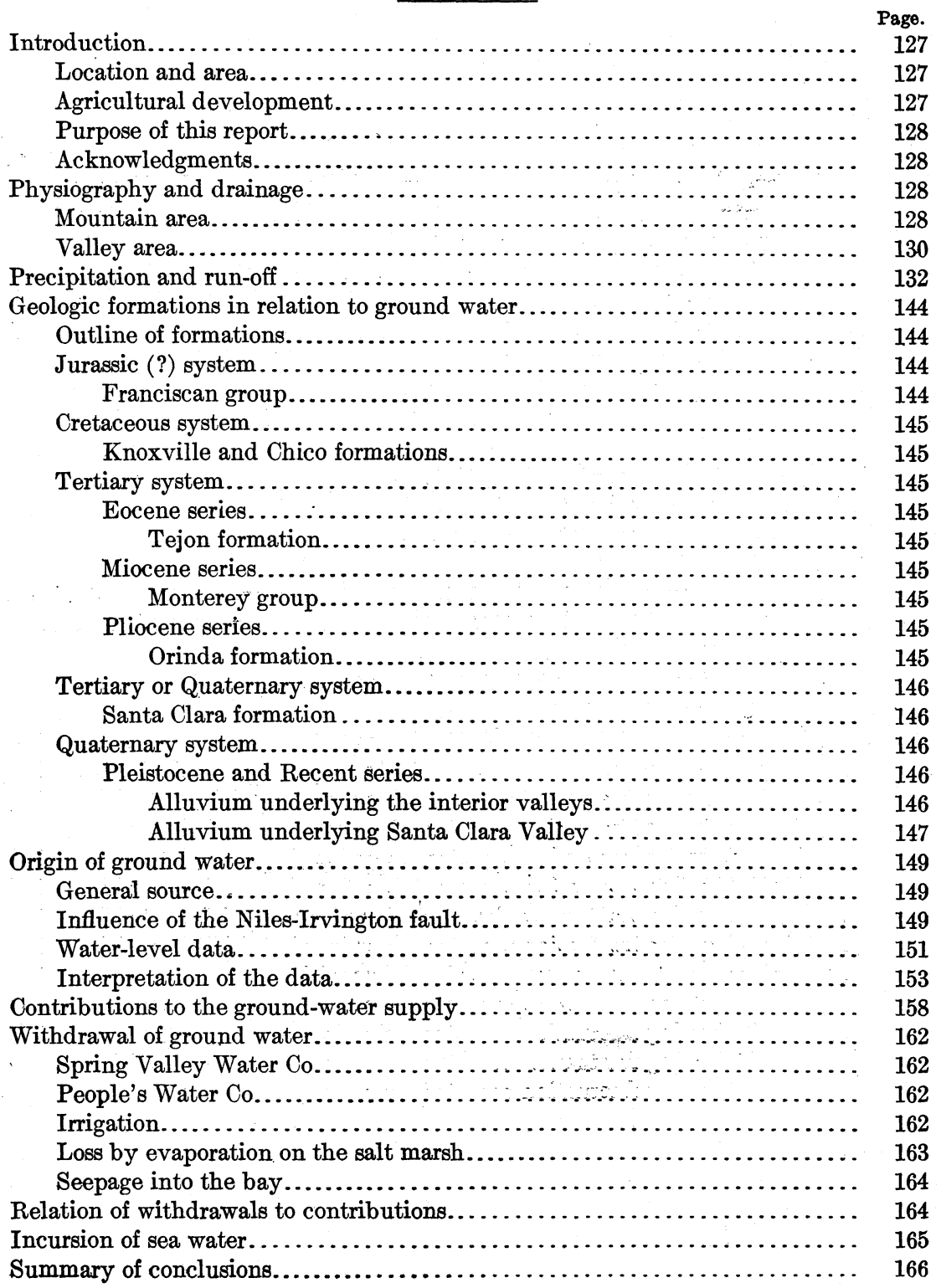




\section{ILLUSTRATIONS.}

Plate IX. Map of the Alameda Creek drainage basin, Cal., and adjacent

Page.

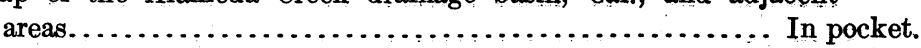

$X$. Map of the Niles cone, Cal., and adjacent areas............ In pocket.

XI. $A$, View of Niles cone, Cal., showing its regular topography and slight gradient; $B$, Niles-Irvington fault scarp, Cal., with The Lagoon in the foreground.

XII: Notch in fault scarp at Tule Pond, Cal., representing ancient stream channel cut off by the displacement........................

XIII. $A$ and $B$, Ancient drainage channel broken by displacement along Niles-Irvington fault, Cal.............................. 133

XIV. Well sections along line O-P, Plate X ................... 148

XV. Well sections along line Q-R, Plate X.................. 148

XVI. Well sections along line S-T, Plate X..................... 148

XVII. Well sections along line U-V, Plate X.................... 148

Figure 9. Map showing submerged stream channels in San Francisco Bay, Cal. 131

10. Diagram showing effect of topography on rainfall in California and

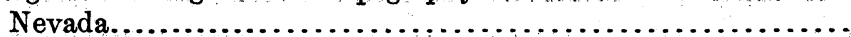

11. Diagram showing effect of topography on rainfall in the Coast Range of California.

12. Profiles of water table along line $F-G$, Plate $X . \ldots \ldots \ldots \ldots \ldots \ldots .150$

13. Graph showing fluctuation of water table along line F-G, Plate X.. $\quad 150$.

14. Graph showing fluctuation of water table along line A-B, Plate X.. 153

15. Graph showing fluctuation of water table along line A-C, Plate X.. 153

16. Graph showing fluctuation of water table along line D-E, Plate X.. 154

17. Graph showing fluctuation of water table along line H-E, Plate X.. 155

18. Graph showing fluctuation of water table along line I-J, Plate X.. 155

19. Graph showing fluctuation of water table along line K-L, Plate X.. 156

20. Profiles of water table along line M-N, Plate X .............. 157

21. Graph showing fluctuation of water table along line $\mathrm{C}_{1}$, Plate X... 158

22. Graph showing fluctuation of water table along line $\mathrm{C}_{2}$, Plate $\mathrm{X}$... 159

23. Graph showing fluctuation of water table along line $C_{3}$, Plate $X \ldots 160$

24. Graph showing fluctuation of water table along line $\mathrm{C}_{4}$, Plate X.. 160 IV 


\title{
GROUND-WATER RESOURCES OF THE NILES CONE AND ADJACENT AREAS, CALIFORNIA.
}

\author{
By W. O. Clark.
}

\section{INTRODUCTION.}

Location and area.-The area covered in detail by this report comprises the Niles cone and adjacent tracts in Santa Clara Valley, Cal., just east of the south end of San Francisco Bay. The drainage basins of Alameda Creek and other streams tributary to this valley area are also described in respect to the conditions that influence its ground-water supply. (See Pl. IX, in pocket.)

The Niles cone, which is built of the alluvial deposits of Alameda Creek, is bounded on the north by a line that extends from the town of Niles to a point about half a mile north of Alvarado, and on the south by a line that extends from Niles to Jarvis Landing, approximately as shown in Plate IX. The tract between the Niles cone and a line extending from Mission San Jose through Irvington and a point on the Southern Pacific Railroad about 1 mile southeast of Mowry station is physiographically not a part of the cone but is intimately related to it in regard to ground-water supplies. The cone and this adjacent tract are cut by a fault, shown in Plates IX and $\mathrm{X}$ (in pocket), which profoundly influences the ground-water conditions. The cone covers about 11,800 acres, and the part of the additional tract lying west of the fault covers about 9,000 acres, both exclusive of the salt marsh. The estimates of ground water given in this report refer to the 20,800 acres of the cone and that portion of the adjacent tract that lies west of the fault.

Agricultural development.-The region about Niles is one of intensive cultivation. The holdings are for the most part small, being tracts of $5,10,20$, or 40 acres. Nearer the bay the holdings are as a rule considerably larger, many of them comprising from 100 to several hundred acres. Here the cultivation is less intensive, the crops being largely oats, barley, and alfalfa. In many places near the salt marsh these crops give way to pasture lands. The acreage of alfalfa is rapidly increasing, and in a short time much of the oat and barley land will probably be converted into alfalfa fields. This change will increase the amount of water needed for irrigation. 
The areas covered by different crops in 1913 were plotted and measured for the Niles cone and a small additional area and were estimated for the remaining part of the related tract west of the fault shown on Plate X. The acreage of the different crops west of the fault thus determined is as follows: Orchard, 2,900 acres; tomatoes, 1,350 acres; potatoes, 380 acres; other vegetables, 1,300 acres; corn, 340 acres; sugar beets, 75 acres; strawberries, 65 acres; peas, 25 acres; nursery, 550 acres; alfalfa, 2,430 acres; oats and barley, 5,170 acres; and pasture, 5,560 acres.

Purpose of this report.-For more than 20 years there bas been contention between the people in the vicinity of Niles and the water companies that take large supplies from the Niles cone and tributary drainage basins to San Francisco and other cities on the bay. The farmers of the vicinity have recently been organized into a water district, and further litigation with the water companies is in prospect.

This report is based on an investigation in which an attempt was made to determine the source of the ground water, the quantity now being. withdrawn, the amount available, and the area dependent on Alameda Creek.

Acknowledgments.-Acknowledgments are especially due to $\mathrm{Mr}$. O. E. Meinzer, Dr. J. C. Branner, and Dr. J. P. Smith for much valuable help in the preparation of this report. The writer is also indebted to the Spring Valley Water Co., the People's Water Co., and the Southern Pacific Co., for much valuable information, also to Mr. J. H. Forbes, who ran levels and kept records of many wells for the Spring Valley Water Co., and to the many residents of the district for their kind cooperation in the collection of well records, without which much of the detail of this report could not have been presented:

\section{PHYSIOGRAPHY AND DRAINAGE.}

\section{MOUNTAIN AREA.}

Northeast of the Santa Clara Valley lie the Contra Costa and Mount Hamilton ranges, which have an average elevation of approximately 2,000 feet above sea level and in Copernicus Peak reach an altitude of 4,372 feet. Among the mountains back of the steep front that faces the Santa Clara Valley lie a number of open valleys, which, although not as large as the Santa Clara Valley, are of considerable size. About 640 square miles of this region of mountains and interior valleys is drained by Alameda Creek, which emerges from the mountains at the town of Niles and discharges upon the Niles cone. (See Pl. IX.) Smaller mountain areas are drained by several small streams or canyons to the valley tracts that lie adjacent to the Niles cone. Dry Creek, northwest of Alameda Creek, has a drainage area of approximately 9 square miles; Mission Creek, south- 
east of Alameda Creek, has a drainage area somewhat less than that of Dry Creek. Mission Creek and the smaller streams between it and Alameda Creek have furnished the sediments that underlie the area south of the Niles cone and west of the fault line.

The principal interior valleys within the Alameda Creek basin are the Livermore Valley, with its two arms-Amador and Las Positas valleys - the Sunol Valley, the Calaveras Valley, and the Isabel Valley. The Livermore Valley is larger than the others combined. These are all open valleys, surrounded by mountains. They apparently owe their existence in large part to the extensive faulting and other earth movements by which this region has been affected in recent geologic time.

The Alameda Creek drainage system comprises two main branches which, flowing almost directly toward each other, join in the vicinity of Sunol, and thence discharge their united waters through a canyon that is about 6 miles long and that has its outlet at Niles. The south branch, which carries the name of Alameda Creek, receives the drainage of Calaveras Creek, San Antonio Creek, and several smaller streams. The north branch, known as Arroyo de la Laguna, is the outlet of the Livermore Valley, into which discharge several relatively large streams, most important of which are Arroyo del Valle and Arroyo Mocho.

Alameda Creek rises on the northern slopes of Packard Ridge, a few miles north of Mount Hamilton and just east of Rattlesnake Butte, which has an elevation of 3,444 feet above sea level.

Calaveras Creek has a drainage area of approximately 100 square miles. Its principal tributary, Arroyo Hondo, is formed by the junction of Isabel and Smith creeks. Isabel Creek rises near the boundary line between Santa Clara and Stanislaus counties, flows through Isabel Valley, passes round Mount Hamilton on the east and north, and joins Smith Creek about 4 miles northwest of Mount Hamilton. Smith Creek rises on the western slopes of Pine Ridge, at an elevation of about 3,400 feet, and passes west of Mount Hamilton, to its junction with Isabel Creek. From the junction Arroyo Hondo leads to the lower end of Calaveras Valley, where it discharges into Calaveras Creek. The united waters then pass through about a mile of steep-walled canyon and discharge into Alameda Creek. Although Arroyo Hondo is said to discharge into Calaveras Creek, the water carried by the Calaveras above the confluence is practically negligible.

San Antonio Creek, which discharges into Alameda Creek from the east, a short distance above Sunol, has a drainage area that covers about 35 square miles and lies at elevations ranging from 500 to 3,600 feet above sea level. 
Arroyo del Valle, which discharges, through the southern part of Livermore Valley, into Arroyo de la Laguna, receives the drainage of about 150 square miles-the largest single unit in the Alameda basin. Its headwaters are on the borders of Santa Clara and Stanislaus counties, 2,500 to 3,000 feet above sea level.

Arroyo Mocho, which also discharges into the Livermore Valley, has a drainage area of approximately 50 square miles. It has its source about the four corners of Alameda, San Joaquin, Stanislaus, and Santa Clara counties. For most of its course it flows through a region that is from 2,500 to 3,000 feet above sea level.

Among the other streams discharging into the Livermore Valley, the largest are Arroyo las Positas and Arroyo Seco.

\section{VALLEY AREA.}

In contrast to the rugged mountain area above described the Santa Clara Valley appears exceedingly flat and monotonous. (See Pl. XI, A.) Yet it has its own features, which, although not so striking as those of the mountain area, are quite as distinctive and significant. The valley area covered in this report consists essentially of the Niles cone and the alluvial cones or fans of Mission Creek and other small streams. The slope of all these alluvial cones is very gentle, being on an average only about 10 feet to the mile. The gentleness of the slope shows that water has been abundant during the process of alluvial filling, for cones built under conditions of meager water supply are invariably steeper, many of them having slopes of as much as 500 feet to the mile.

Among the several alluvial cones in this vicinity the Niles cone is small in comparison with the relatively large drainage basin tributary to it, but this condition is due partly to the fact that the materials of the cone were derived largely from the 15 to 20 square miles of the Alameda Creek drainage basin lying southwest of Livermore Valley, most of the alluvial material derived from the remaining 620 or 625 square miles of the basin being left behind in the Livermore, Sunol, and other valleys.

A conspicuous and significant topographic feature in this nearly flat area is an escarpment formed by recent faulting. This escarpment, which faces the mountains and trends $\mathrm{N} .35^{\circ} \mathrm{W}$., crosses a reentrant of the valley and cuts off the apex of the Niles cone as well as some of the flat land to the south. It begins in the vicinity of Tule Pond and extends past The Lagoon to the town of Irvington. It ranges in height from only a few feet to 20 or 25 feet at its highest point, at The Lagoon (Pls. X and XI, B). A distinct ridge approaches this escarpment from the west in the vicinity of The Lagoon, as if there had been some thrusting force and a slight buckling at the time of faulting. Behind this escarpment are two ponds-The Lagoon, 


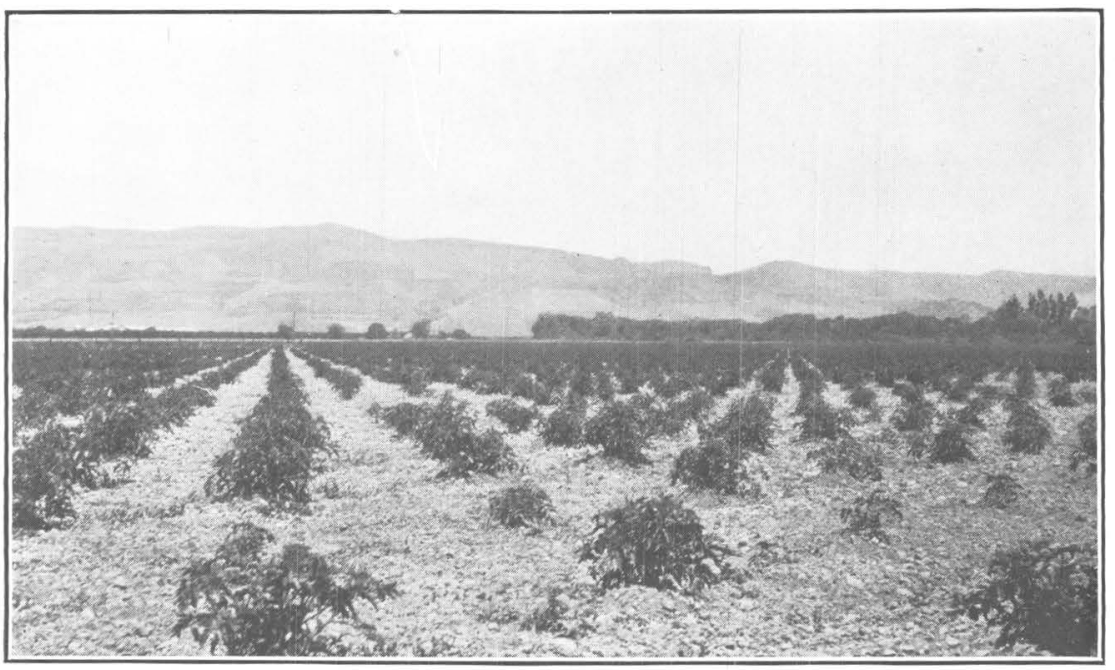

A. VIEW OF NILES CONE, CAL., SHOWING ITS REGULAR TOPOGRAPHY AND SLIGHT GRADIENT.

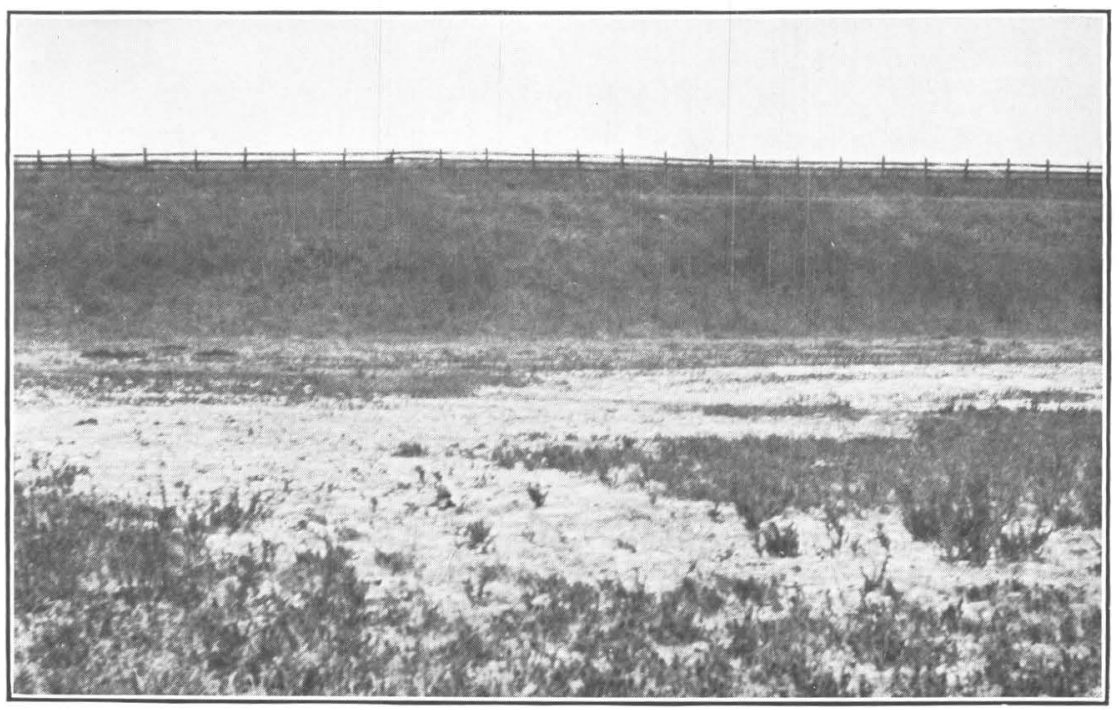

$B$. NILES-IRVINGTON FAULT SCARP, CAL., WITH THE LAGOON IN THE FOREGROUND. 
which when full has an area of 200 to 300 acres, and Tule Pond, which probably at no time covers more than 50 acres. These ponds were never known to be entirely dry until the summer of 1913, near the close of the two-year period having a lighter precipitation than any other

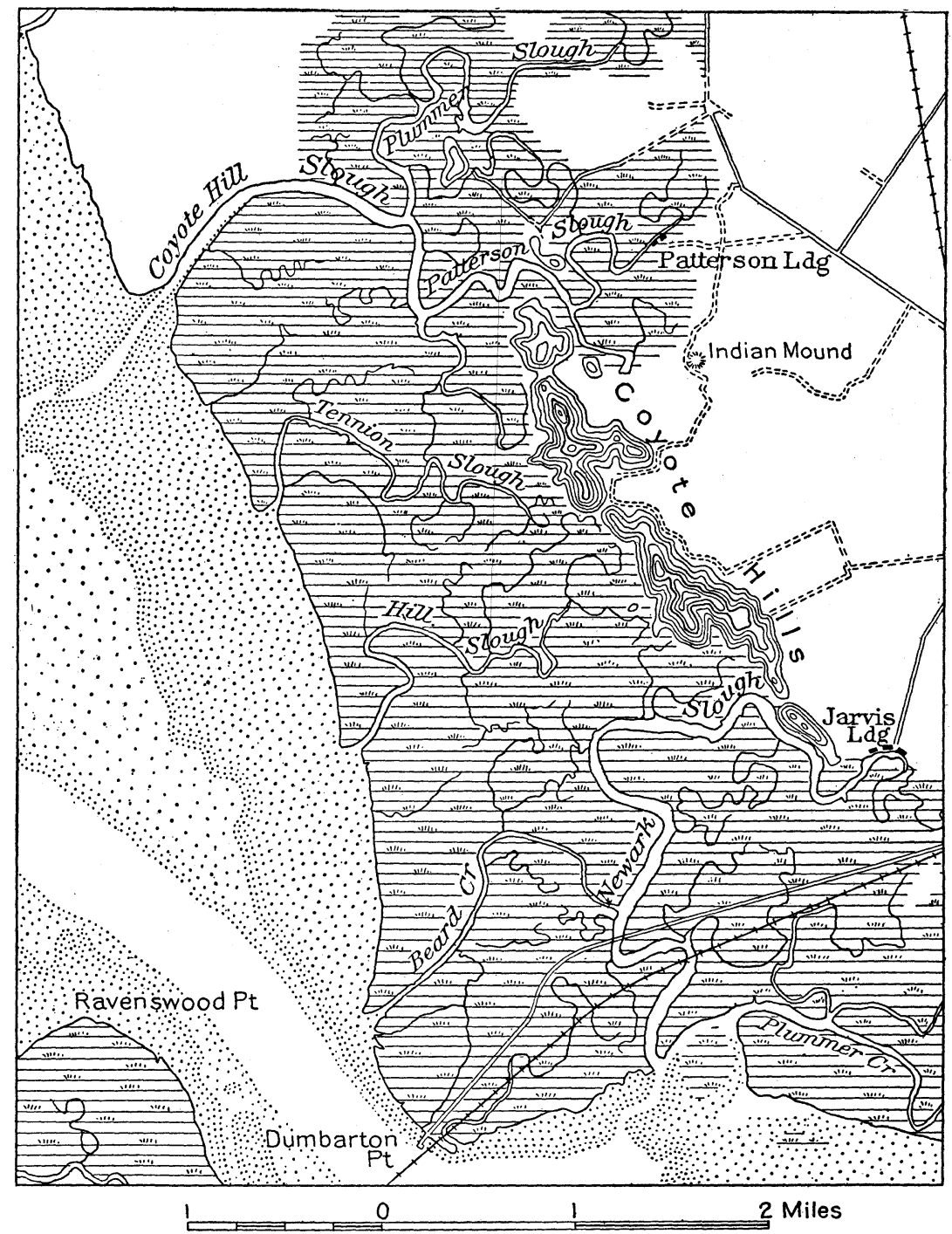

Figure 9.-Map showing submerged stream channels in San Francisco Bay, Cal. (From Coast and Geodetic Survey.)

two consecutive years on record. They are, however, always shallow and vary greatly in extent with the season. Southwest of the ponds rises the relatively abrupt fault scarp, but to the northeast there is no perceptible bank except at the northern shore of Tule Pond, where 
there is a small cliff that is probably due partly to erosion by water entering at that end and partly to wave action. On the side toward the mountains the land rises very gradually to the steep front of the mountains.

This fault scarp has caused considerable change in drainage. (See Pl. X.) The so-called Laguna drainage basin, which should not be confused with the Arroyo de la Laguna that forms the outlet of the Livermore Valley, consists of all of the area tributary to The Lagoon and comprises several small units south of Alameda Creek. (See Pl. IX.) This drainage basin is now limited on the west by the fault scarp, but before the recent movement along the fault its waters discharged over the flat land west of the fault and south of the Niles cone and supplied the sediments underlying this land. Mission Creek, coming from the hills, flows northwestward into The Lagoon, but the stream that forms the outlet of The Lagoon has a southeasterly course and makes an angle of only about $60^{\circ}$ with Mission Creek. A notch in the escarpment, shown in Plate XII, represents an abandoned stream channel (Pl. XIII, $A$ ), which is distinctly marked from this point to the place where it enters the salt marsh, just south of the Little Coyote Hills. In Plate XIII, $B$, is shown a similar ancient stream channel which is not so sharply defined as this one but is nevertheless distinct. It starts at a notch in the escarpment near the north end of The Lagoon and can be traced to a point about a mile southwest of Newark, beyond which it has been artificially straightened. Both these ancient drainage lines are shown on Plate X.

At the foot of the Niles cone and extending somewhat farther south are two groups of rock hills known as the Coyote Hills and the Little Coyote Hills, which are separated from each other by a gap of salt marsh only about half a mile wide. (See Pls. IX and X.) The Coyote Hills reach an elevation of a little more than 290 feet, and the Little Coyote Hills only about 50 feet. The salt marsh gap separating these two groups of hills is the inlet of Newark Creek, or the outlet of Sanjen de los Alisos, which at no very remote date was the main Alameda Creek. Its old channel is traceable for some distance beneath the waters of the bay. Coyote Hill Creek, at the north end of the Coyote Hills, is also an ancient outlet of Alameda Creek, and its channel is distinctly traceable for a mile or more beneath the bay. (See fig. 9.)

\section{PRECIPITATION AND RUN-OFF.}

The Contra Costa and Mount Hamilton ranges are effective rain producers. They lie at right angles to the path of the prevailing moisture-laden winds from the Pacific and are of sufficient height to cause considerable condensation from these winds. Though their rainfall is less than that of the humid parts of the United States it 


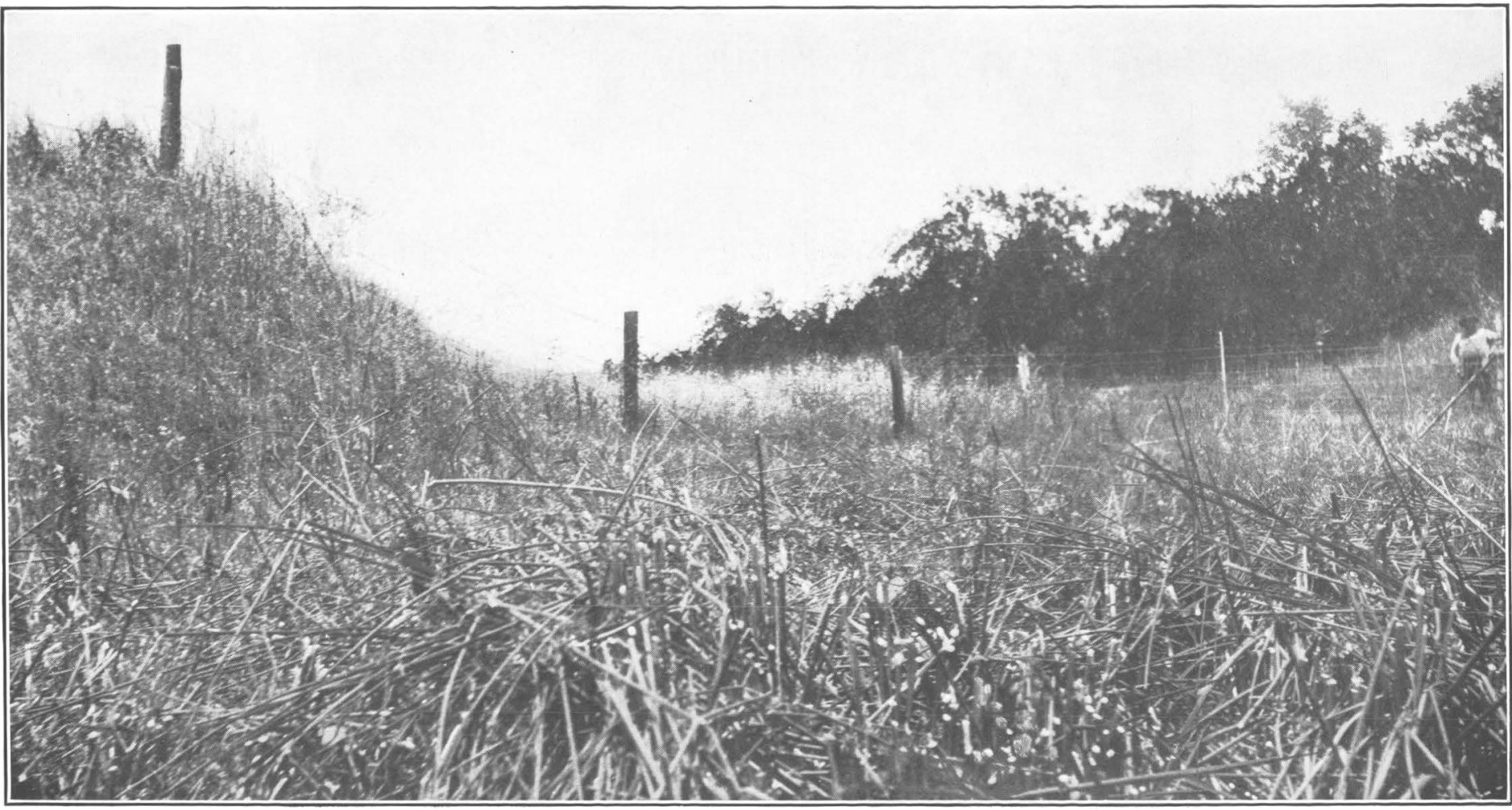

NOTCH IN FAULT SCARP AT TULE POND, CAL., REPRESENTING ANCIENT STREAM CHANNEL CUT OFF BY THE DISPLACEMENT. 


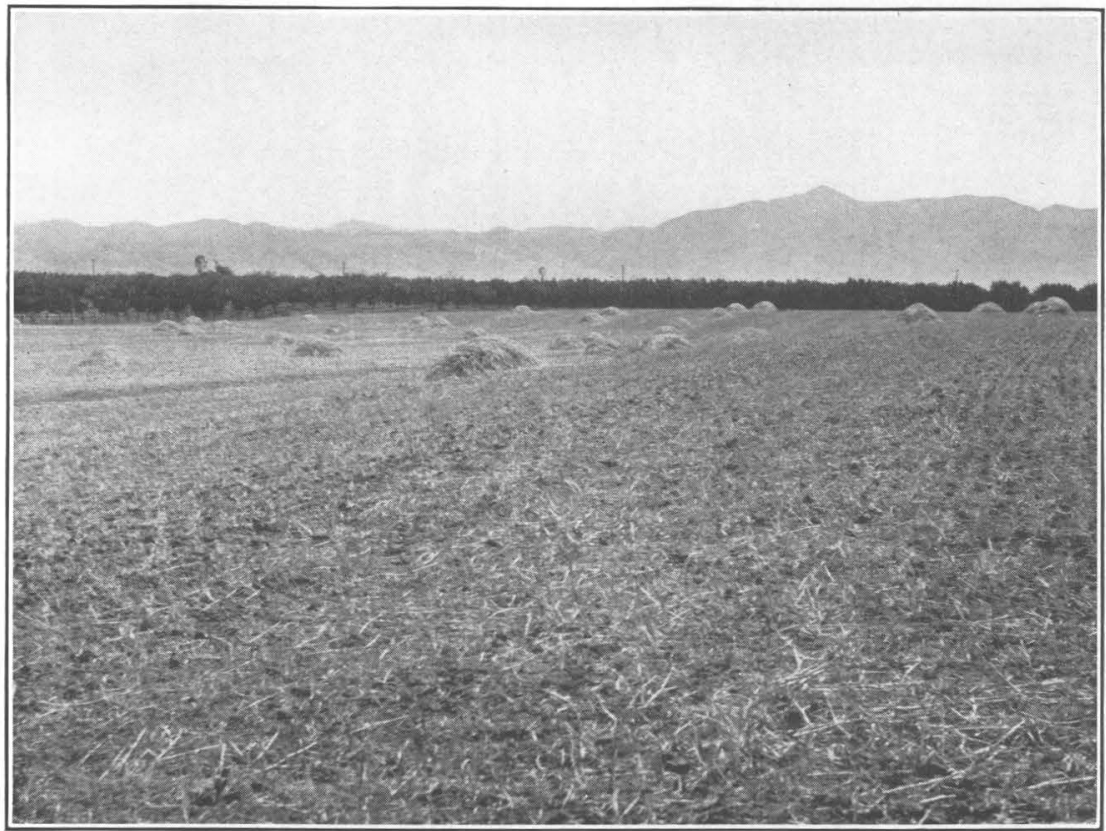

A.

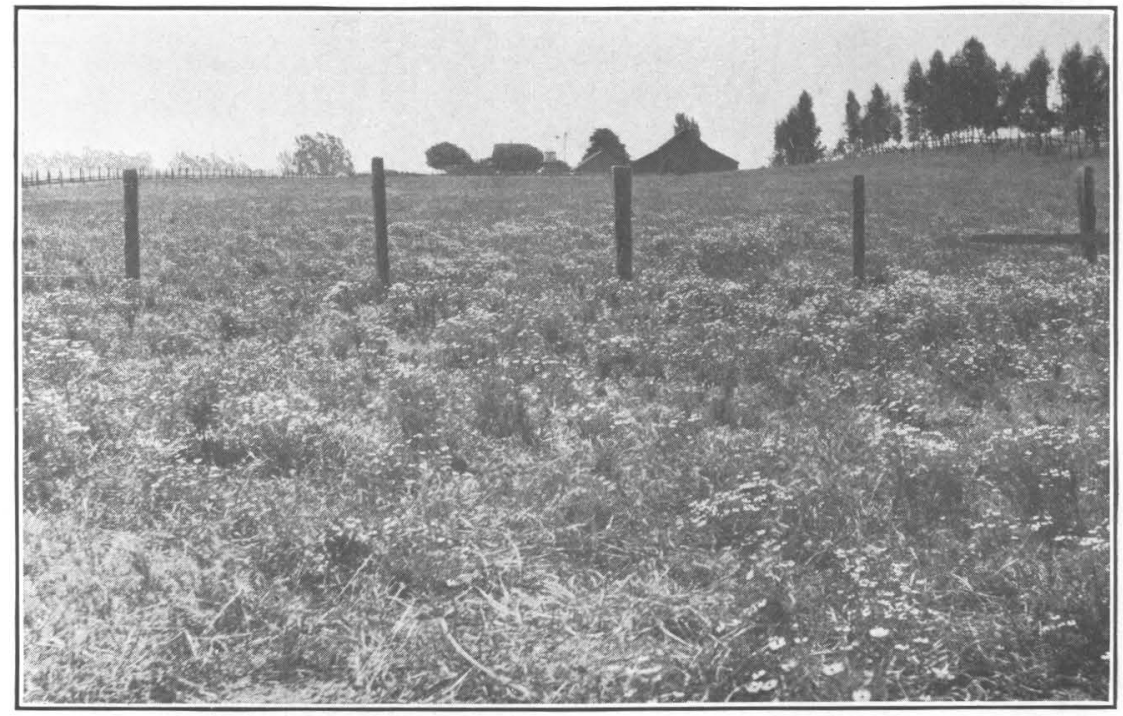

B.

ANCIENT DRAINAGE CHANNEL BROKEN BY DISPLACEMENT ALONG NILES-IRVINGTON FAULT, CAL. 
is heavy at certain times during the winter, when the air coming from the ocean nearly saturated is forced over the mountains and thereby cooled. Consequently Alameda Creek is subject to heavy floods.

There is a marked increase in rainfall with altitude. McAdie, ${ }^{1}$ quoting records obtained in the vicinity of Fresno, Cal., gires the

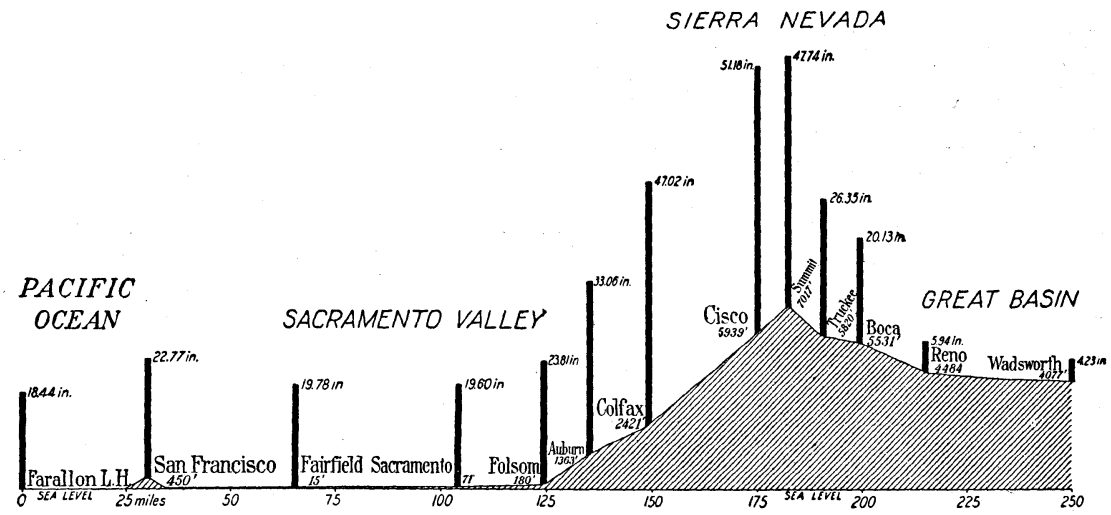

FIGURE 10.-Diagram showing effect of topography on rainfall in California and Nevada. After Hamlin

rate of increase as 1.18 inches of rainfall to 100 feet of altitude, and he calculates from further data for the season of 1911-12 a difference of 1 inch in 100 feet. $^{2}$ Hamlin ${ }^{3}$ shows the effect of topography on rainfall by means of the diagrams given in figures 10 and 11 .

On Mount Hamilton, 4,209 feet above sea level, the average annual precipitation, according to United States Weather Bureau records,

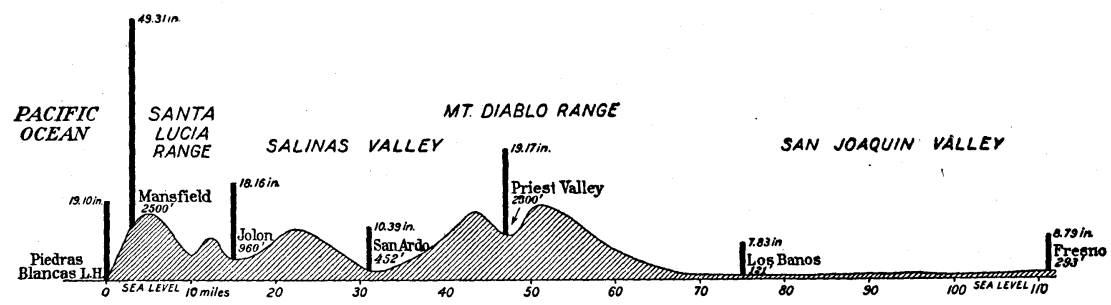

FIGURE 11.-Diagram showing effect of topography on rainfall in the Coast Range of California. After Hamlin.

is 31.42 inches (p. 137). Twice in January, 1911, there were heavy downpours on this mountain. During one of these storms 9.19 inches fell in 24 hours and during the other 5.56 inches fell in an equal time. ${ }^{4}$ Arroyo Hondo, which drains all sides of the lofty mountain mass that culminates in Mount Hamilton and Copernicus

1 McAdie, A. G., U. S. Dept. Agr. Monthly Weather Review, vol. 39, No. 9, September, 1911.

2 McAdie, A. G., Rainfall of California: California Univ. Pubs., vol. 1, No. 4, p. 158, Feb. 19, 1914.

3 Hamlin, Homer, Water resources of the Salinas Valley, California: U. S. Geol. Survey Water-Supply Paper 89, pp. 41, 42, 1904.

1 McAdie, A. G., Rainfall of California: California Univ. Pubs., vol. 1, No. 4, p. 179, Feb. 19, 1914. 
Peak and also other large areas with high altitudes, is the best water producer in the Alameda system. According to records of the Spring Valley Water Co. ${ }^{1}$ the annual discharge of Arroyo Hondo at the Calaveras dam site, in the canyon below Calaveras Valley, for the five consecutive seasons from 1898-99 to 1902-3, was 15,535 million gallons, or 47,675 acre-feet; and for the nine seasons for which records $^{2}$ are available almost 19,689 million gallons, or 60,423 acre-feet. This 9-year record is not a continuous one, there being a break of one year between the 5-year period 1898-1903 and the 4-year period 1904-1908.

The only available records of the discharge of San Antonio Creek are those from January to June, inclusive, 1912, given by the Spring Valley Water Co. ${ }^{3}$ The total discharge during this period was reported to be 454 million gallons, or about 1,393 acre-feet. According to records of the Southern Pacific Co. and the Spring Valley Water Co. the mean annual rainfall at Sunol during a period of 23 years was 22.26 inches. The minimum for any one season was 10.5 inches (1897-98) and the maximum 38.99 inches (1889-90).

The meager records of rainfall in the region tributary to Arroyo del Valle range from 18 to 43 inches a year. The Spring Valley Water Co., ${ }^{4}$ by its method of interpolation (for the correctness of which the writer does not vouch), estimates the mean annual rainfall for the entire Arroyo del Valle drainage area at 20.80 inches. The average annual run-off at the dam site of the Spring Valley Water Co., about $3 \frac{1}{2}$ miles above the point where Arroyo del Valle enters the Livermore Valley, during a period of four seasons, from 1904-5 to $1907-8$, inclusive, as computed by the writer from data given by the company, ${ }^{2}$ was 14,050 million gallons, or 43,118 acre-feet.

There are no records of rainfall in the area tributary to Arroyo Mocho. At Livermore, 487 feet above sea level, the Government records show the average annual rainfall for 42 years to be $\mathbf{1 5 . 4 9}$ inches (p. 138), but the average altitude of the area tributary to Arroyo Mocho is much greater than that of Livermore, and its rainfall is therefore heavier. According to the records of the Southern Pacific Co. the average annual rainfall at Pleasanton during a period of eight years was 16.03 inches (p. 139). A 13-year record of the Spring Valley Water Co. ${ }^{5}$ for a point about 2 miles southeast of Pleasanton gives the average annual rainfall as 25.21 inches.

\footnotetext{
1 The future water supply of San Francisco from the conservation and use of its present resources, p. 323, Oct. 31, 1912.

2 Idem, p. 113.

3 Idem, p. 342.

4 Idem, p. 284a.

5 Idem, p. 292.
} 
In the following tables are given the available records of precipitation at stations in or near the Alameda Creek drainage basin and the Spring Valley Water Co.'s records of discharge of Alameda Creek at the Sunol dam, and also the discharge over Brightside weir. The Brightside weir is situated in Alameda Canyon, between 1 and 2 miles below the Sunol dam. The water flowing over this weir is ground water that has been pumped by the Spring Valley Water Co. and is on its way to San Francisco. Because this water would rise, in large part, and flow through Alameda Creek to the Niles cone it has been added to the flow of that creek in the table on page 143. The discharge records were generously furnished to the United States Geological Survey by the Spring Valley Water Co.

Records of precipitation in or near the Alameda Creek drainage basin (in inches).

Alvarado, Alameda County. $a$

[Elevation 19.5 feet.]

\begin{tabular}{|c|c|c|c|c|c|c|c|c|c|c|c|c|c|}
\hline Season. & July. & Aug. & Sept. & Oct. & Nov. & Dec. & Jan. & Feb. & Mar. & Apr. & May. & June. & $\begin{array}{c}\text { Total } \\
\text { for } \\
\text { season. }\end{array}$ \\
\hline 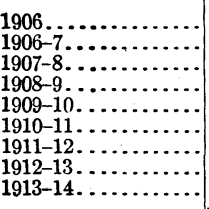 & $\begin{array}{r}0 \\
0 \\
0 \\
b 0 \\
0 \\
0 \\
0 \\
0\end{array}$ & $\begin{array}{l}0 \\
0.05 \\
0 \\
b 0 \\
0 \\
0 \\
0 \\
0 \\
\end{array}$ & $\begin{array}{l}0.26 \\
.03 \\
.10 \\
.90 \\
0 \\
0 \\
0 \\
0\end{array}$ & $\begin{array}{l}0 \\
.67 \\
0 \\
1.90 \\
.85 \\
.23 \\
.50 \\
0\end{array}$ & $\begin{array}{l}1.22 \\
1.02 \\
1.58 \\
.50 \\
1.23 \\
1.23 \\
3.94\end{array}$ & $\begin{array}{r}7.57 \\
4.85 \\
1.63 \\
4.94 \\
.72 \\
1.91 \\
.99 \\
2.37\end{array}$ & $\begin{array}{r}4.27 \\
3.27 \\
9.28 \\
2.63 \\
12.15 \\
4.94 \\
2.96 \\
8.81\end{array}$ & $\begin{array}{r}3.42 \\
3.06 \\
5.83 \\
1.08 \\
2.35 \\
.35 \\
.20 \\
2.87\end{array}$ & $\begin{array}{l}8.12 \\
1.13 \\
2.97 \\
2.86 \\
4.28 \\
2.73 \\
1.28 \\
1.05\end{array}$ & $\begin{array}{r}0.85 \\
.12 \\
1.10 \\
0 \\
.47 \\
.74 \\
1.66 \\
.59 \\
.73\end{array}$ & $\begin{array}{l}1.09 \\
0 \\
.40 \\
0 \\
0 \\
.38 \\
1.16 \\
.60 \\
.10\end{array}$ & $\begin{array}{l}0.59 \\
.74 \\
0 \\
b .29 \\
0 \\
0 \\
.67 \\
.0 \\
.48\end{array}$ & $\begin{array}{r}23.72 \\
14.58 \\
b 21.14 \\
b 16.36 \\
21.97 \\
13.65 \\
8.35 \\
20.35\end{array}$ \\
\hline Mean.................. & 0 & 0 & .16 & .52 & 1.19 & 2.87 & 6.04 & 2.40 & 3.05 & .69 & .41 & b. 31 & $b 17.64$ \\
\hline
\end{tabular}

Alviso, Santa Clara County. $a$

[Elevation 11.4 feet.]

\begin{tabular}{|c|c|c|c|c|c|c|c|c|c|c|c|c|c|}
\hline Season. & July. & Aug. & Sept. & Oct. & Nov. & Dec. & Jan. & Feb. & Mar. & Apr. & May. & June.I & $\begin{array}{l}\text { Total } \\
\text { for } \\
\text { season. }\end{array}$ \\
\hline 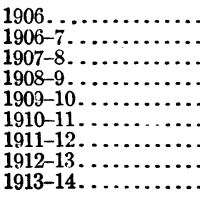 & $\begin{array}{c}0 \\
0 \\
0 \\
h_{0} \\
0 \\
0 \\
0 \\
.07\end{array}$ & $\begin{array}{c}0 \\
0 \\
0 \\
b 0 \\
0 \\
0 \\
0 \\
.03\end{array}$ & $\begin{array}{l}0.20 \\
0 \\
.07 \\
1.02 \\
0 \\
0 \\
.58 \\
0^{.58}\end{array}$ & $\begin{array}{l}0 \\
.30 \\
. \\
.25 \\
.07 \\
.94 \\
0 \\
0\end{array}$ & $\begin{array}{r}0.90 \\
.10 \\
1.23 \\
1.50 \\
.25 \\
.26 \\
.72 \\
3.13\end{array}$ & $\begin{array}{r}5.51 \\
3.42 \\
1.66 \\
4.03 \\
.95 \\
1.90 \\
.50 \\
1.52\end{array}$ & $\begin{array}{r}3.87 \\
1.71 \\
7.75 \\
4.18 \\
11.28 \\
1.47 \\
2.47 \\
6.60\end{array}$ & $\begin{array}{r}2.45 \\
2.49 \\
5.95 \\
1.00 \\
3.11 \\
.33 \\
.18 \\
3.40\end{array}$ & $\begin{array}{r}7.32 \\
.97 \\
2.39 \\
b 3.32 \\
4.88 \\
2.47 \\
1.35 \\
.90\end{array}$ & $\begin{array}{r}0.45 \\
.28 \\
.12 \\
0 \\
6.39 \\
.50 \\
1.00 \\
.35 \\
.40\end{array}$ & $\begin{array}{l}0.70 \\
.20 \\
.37 \\
0 \\
0 \\
.34 \\
.51 \\
.62 \\
.24\end{array}$ & $\begin{array}{l}0.50 \\
.40 \\
0 \\
b .23 \\
.02 \\
.10 \\
.59 \\
0.15\end{array}$ & $\begin{array}{r}20.95 \\
8.09 \\
b 20.28 \\
b 15.71 \\
21.48 \\
9.47 \\
6.77 \\
16.44\end{array}$ \\
\hline Mean........... & .01 & 0 & .23 & .17 & 1.01 & 2.44 & 4.92 & 2.36 & 2.95 & .39 & .33 & .22 & 15.01 \\
\hline
\end{tabular}


Records of precipitation in or near the Alameda Creek drainage basin (in inches)-Contd.

Calaveras, Alameda County. a

[Elevation 600 feet.]

\begin{tabular}{|c|c|c|c|c|c|c|c|c|c|c|c|c|c|}
\hline Season. & July. & Aug. & Sept. & Oct. & Nov. & Dec. & Jan. & Feb. & Mar. & Apr. & May. & June. & $\begin{array}{l}\text { Total } \\
\text { for } \\
\text { season. }\end{array}$ \\
\hline 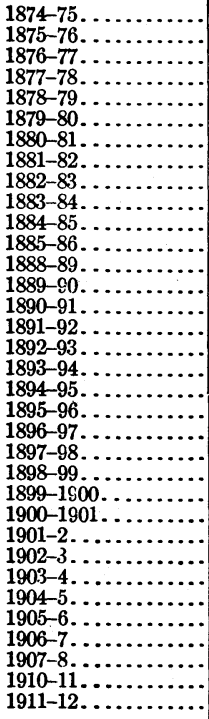 & $\begin{array}{l}0 \\
0 \\
0 \\
0 \\
0 \\
0 \\
0 \\
0 \\
0 \\
0 \\
0 \\
0 \\
0 \\
0 \\
0 \\
0 \\
0 \\
0 \\
0 \\
0 \\
0 \\
0 \\
0 \\
0 \\
0 \\
0 \\
0 \\
0 \\
0 \\
0 \\
0 \\
0 \\
0 \\
0\end{array}$ & $\begin{array}{l}0 \\
0 \\
0 \\
0 \\
0 \\
0 \\
0 \\
0 \\
0 \\
0 \\
.10 \\
0 \\
0 \\
0 \\
0 \\
0 \\
0 \\
0 \\
0 \\
0 \\
.55 \\
0 \\
0 \\
0 \\
0 \\
0 \\
0 \\
0 \\
.62 \\
0 \\
0 \\
0 \\
0 \\
0\end{array}$ & $\begin{array}{l}0 \\
0 \\
0 \\
0 \\
.25 \\
0 \\
0 \\
.21 \\
.46 \\
.33 \\
.25 \\
.10 \\
.50 \\
0 \\
.75 \\
.50 \\
0 \\
.15 \\
1.83 \\
.31 \\
.30 \\
0 \\
.61 \\
0 \\
0 \\
.80 \\
0 \\
0 \\
3.27 \\
0 \\
.33 \\
0 \\
0 \\
.01\end{array}$ & $\begin{array}{l}4.10 \\
.35 \\
.83 \\
.78 \\
1.35 \\
.04 \\
.43 \\
1.88 \\
1.73 \\
1.80 \\
0 \\
0 \\
5.61 \\
0 \\
.30 \\
1.75 \\
.30 \\
3.27 \\
.87 \\
2.02 \\
1.51 \\
1.32 \\
5.68 \\
4.16 \\
1.75 \\
1.80 \\
.18 \\
2.41 \\
0 \\
0 \\
.86 \\
.50 \\
1.26\end{array}$ & $\begin{array}{r}4.57 \\
11.05 \\
.15 \\
1.23 \\
.37 \\
2.99 \\
.69 \\
1.83 \\
2.19 \\
.84 \\
.02 \\
10.95 \\
4.17 \\
3.99 \\
.10 \\
.75 \\
8.39 \\
2.72 \\
1.00 \\
2.36 \\
4.97 \\
1.11 \\
1.08 \\
5.04 \\
7.11 \\
2.01 \\
4.25 \\
5.18 \\
1.73 \\
2.88 \\
1.16 \\
.12 \\
.38 \\
1.01\end{array}$ & $\begin{array}{r}0.18 \\
4.82 \\
0 \\
2.39 \\
.43 \\
5.33 \\
10.64 \\
3.60 \\
2.81 \\
1.13 \\
11.98 \\
2.67 \\
3.32 \\
12.88 \\
4.91 \\
10.29 \\
7.08 \\
3.59 \\
12.02 \\
2.66 \\
4.47 \\
2.45 \\
2.16 \\
3.50 \\
2.15 \\
1.67 \\
1.79 \\
1.31 \\
3.23 \\
1.95 \\
8.60 \\
6.40 \\
.96 \\
3.03\end{array}$ & $\begin{array}{r}5.69 \\
6.82 \\
6.50 \\
10.24 \\
4.66 \\
3.41 \\
4.60 \\
2.34 \\
1.17 \\
5.51 \\
2.91 \\
8.11 \\
.64 \\
10.02 \\
.90 \\
.99 \\
5.71 \\
8.12 \\
9.13 \\
8.41 \\
2.64 \\
1.64 \\
4.53 \\
3.35 \\
4.19 \\
1.58 \\
6.15 \\
1.73 \\
3.20 \\
8.64 \\
5.48 \\
3.10 \\
14.94 \\
2.41\end{array}$ & $\begin{array}{r}0.59 \\
6.80 \\
.67 \\
14.88 \\
3.04 \\
3.00 \\
4.90 \\
2.91 \\
1.94 \\
9.81 \\
.29 \\
1.37 \\
1.07 \\
5.73 \\
6.24 \\
2.19 \\
3.86 \\
9.64 \\
4.02 \\
.52 \\
7.61 \\
3.31 \\
0 \\
1.45 \\
6.69 \\
7.24 \\
2.09 \\
7.94 \\
3.78 \\
3.90 \\
2.13 \\
3.54 \\
3.71 \\
.39\end{array}$ & \begin{tabular}{|c|}
1.15 \\
8.55 \\
1.38 \\
0 \\
5.79 \\
2.30 \\
2.22 \\
7.06 \\
4.33 \\
9.30 \\
.47 \\
2.02 \\
6.63 \\
3.46 \\
3.29 \\
5.01 \\
8.35 \\
2.63 \\
3.00 \\
2.44 \\
7.47 \\
.91 \\
9.83 \\
2.83 \\
1.88 \\
5.25 \\
7.60 \\
6.89 \\
5.68 \\
6.77 \\
14.41 \\
1.13 \\
6.01 \\
3.12
\end{tabular} & $\begin{array}{l}0 \\
1.75 \\
.62 \\
0 \\
3.54 \\
8.24 \\
1.35 \\
3.48 \\
3.16 \\
6.87 \\
1.47 \\
6.35 \\
1.06 \\
2.10 \\
2.89 \\
1.81 \\
3.15 \\
1.05 \\
2.75 \\
6.53 \\
.90 \\
.50 \\
1.28 \\
2.80 \\
2.76 \\
1.95 \\
.85 \\
4.01 \\
1.47 \\
1.52 \\
.24 \\
.26 \\
.96 \\
2.02\end{array}$ & $\begin{array}{l}0 \\
.36 \\
0 \\
0 \\
2.48 \\
1.07 \\
.05 \\
.45 \\
3.46 \\
.39 \\
.05 \\
0.48 \\
2.48 \\
1.75 \\
.90 \\
3.20 \\
.91 \\
2.04 \\
1.61 \\
1.72 \\
0 \\
1.67 \\
1.17 \\
1.19 \\
1.72 \\
1.02 \\
.42 \\
.25 \\
3.33 \\
1.61 \\
.01 \\
1.93 \\
.62 \\
.82\end{array}$ & $\begin{array}{l}0 \\
0 \\
0 \\
0 \\
.15 \\
0 \\
.54 \\
.01 \\
0 . \\
1.35 \\
.29 \\
0 \\
0 \\
0 \\
.25 \\
.20 \\
0 \\
.57 \\
0 \\
0 \\
.27 \\
.27 \\
0 \\
0 \\
0 \\
0 \\
0 \\
0 \\
0 \\
.77 \\
.26 \\
0 \\
.04 \\
.72\end{array}$ & $\begin{array}{l}16.28 \\
40.50 \\
12.15 \\
29.02 \\
21.43 \\
27.69 \\
25.03 \\
22.32 \\
21.40 \\
37.26 \\
19.63 \\
31.66 \\
19.87 \\
45.54 \\
20.23 \\
25.24 \\
39.20 \\
30.81 \\
38.63 \\
25.82 \\
31.20 \\
13.37 \\
21.98 \\
25.84 \\
30.66 \\
23.27 \\
24.95 \\
27.49 \\
28.72 \\
28.04 \\
32.98 \\
17.34 \\
28.12 \\
14.79\end{array}$ \\
\hline Mean............. & 0 & .04 & .32 & 1.50 & 2.89 & 4.31 & 4.98 & 4.04 & 4.68 & 2.34 & 1.14 & .17 & 26. 41 \\
\hline
\end{tabular}

a The future water supply of San Francisco from the conservation and use of its present resources, p. 449. Oct. 31, 1912. 
Records of precipitation in or near the Alameda Creek drainage basin (in inches)-Contd.

Lick Observatory, Santa Clara County.a

[Elevation 4,209 feet.]

\begin{tabular}{|c|c|c|c|c|c|c|c|c|c|c|c|c|c|}
\hline Season. & July. & Aug. & Sept. & Oct. & Nov. & Dec. & Jan. & Feb. & Mar. & Apr. & May. & June. & $\begin{array}{c}\text { Total } \\
\text { for } \\
\text { season. }\end{array}$ \\
\hline $1881 \ldots$ & & & & & & & 3.51 & 5.99 & 1.13 & 0.98 & 0.09 & 0.33 & \\
\hline 1881-82. & 0 & 0 & 0.10 & 0.33 & 0.91 & 9.72 & 3. 55 & 2.90 & 5. 40 & 4. 70 & .48 & $1.00 \mathrm{O}$ & 29.15 \\
\hline $1882-83 \ldots \ldots \ldots \ldots$ & 0 & 0 & & 6.16 & 3.45 & 1.93 & 3.10 & 3.75 & 8.66 & 2.66 & 7.55 & 0 & 37.26 \\
\hline $1883-84 \ldots \ldots \ldots \ldots$ & 0 & 0 & .65 & 2.15 & 1. 48 & 2.05 & 5.60 & 12.76 & 16.35 & 1.24 & 3.85 & 3.85 & 58.09 \\
\hline $1884-85 \ldots \ldots \ldots$ & 0 & & .65 & 3.71 & .01 & 33.84 & 1.99 & .57 & 1.15 & 2.08 & .16 & .30 & 44.67 \\
\hline $1885-86 \ldots \ldots \ldots \ldots$ & 0 & 0 & .15 & .05 & 1.92 & 9.80 & 4.44 & 1.80 & 5.77 & 6.79 & .70 & 0 & 31.42 \\
\hline $1886-87 \ldots \ldots \ldots \ldots$ & 0 & 0 & & .60 & 2.82 & 2.34 & 2.83 & 7.80 & 1.39 & 5.75 & .25 & 0.30 & 24.08 \\
\hline $1887-88 \ldots$ & .04 & 0 & .33 & .09 & 0.90 & 11.25 & 10.04 & 1.38 & 3.40 & .68 & 1.25 & 0.67 & 30.03 \\
\hline $1888-89 \ldots \ldots \ldots \ldots$ & & & .49 & .03 & 3.27 & 4.23 & 1.04 & 1. 42 & 6.17 & 1. 92 & 3.21 & 0.05 & 21.85 \\
\hline $1889-90 \ldots$ & 0 & 0 & & 4. 38 & 4. 46 & 13.19 & 7.93 & 6.60 & 4.39 & 1.79 & 2.42 & 0 & 45.16 \\
\hline $1890-91$. & 0 & 0 & .80 & .02 & .58 & 5.39 & 1.38 & 7.12 & 4. 10 & 3.08 & 1.01 & 0.57 & 24.05 \\
\hline 1891-92. & 0 & & .28 & .61 & .38 & 9.54 & 1.97 & 2.99 & 5.98 & 1.90 & 3.52 & .32 & 27.49 \\
\hline $1892-93$. & 0 & Tr. & .24 & 1.38 & 10.30 & 5.56 & 3.29 & 3.45 & 8.99 & 3.61 & .95 & .16 & 37.93 \\
\hline 1893-94. & & & .48 & .66 & 4.01 & 3.58 & 9.74 & 10.52 & 2.54 & .89 & 2.78 & 0.64 & 35.84 \\
\hline 1894-95. & .02 & Tr. & 1.64 & 2.98 & .84 & 11.90 & 10.00 & 3.08 & 1.46 & 2.30 & 2.39 & 0 & 36.61 \\
\hline $1895-96$. & .01 & & .08 & .78 & 2.46 & 3.16 & 9.54 & 1.08 & 3. 83 & 6.70 & 2.10 & .02 & 29.76 \\
\hline $1896-97$. & Tr. & & .47 & 1.85 & 5.86 & 4. 91 & 3.50 & 7. 42 & 6.45 & .82 & .28 & .38 & 32.22 \\
\hline 1897-98. & & & .07 & 1. 25 & 1.51 & 2.70 & 2.30 & 4. 16 & 2.04 & .84 & 2.41 & .38 & 17.66 \\
\hline $1898-99$ & 0 & & .29 & 1.33 & 1.23 & 2.13 & 5.63 & .75 & 11.11 & 1.40 & 1.47 & .39 & 25.73 \\
\hline-1900 . & & .12 & Tr. & 6.37 & 4. 92 & 4.16 & 3. 26 & 1.70 & 3.37 & 4. 06 & 1.35 & Tr. & 29.31 \\
\hline 1900 & .01 & 02 & .08 & 3.48 & 7.76 & 2.21 & 5.76 & 5.92 & 1.98 & 3.33 & 1.07 & .02 & 31.64 \\
\hline $1901-2$ & & .05 & 1.08 & 2. 19 & 2.89 & 1. 61 & 1.44 & 9.42 & 5.19 & 2.61 & 1.14 & & 27.62 \\
\hline 1902-3. & 0 & & & 2.05 & 3.01 & 3.11 & 8.86 & 2.20 & 9.89 & 1.12 & .05 & Tr. & 30.29 \\
\hline $1903-4$. & & & Tr. & .29 & 7. 67 & 1. 39 & 1.98 & 9.53 & 8.06 & 4. 28 & .45 & & 33.78 \\
\hline 1904-5. & Tr. & & 2.33 & 2.51 & 2.05 & 3. 84 & 4.04 & 4. 19 & 5.91 & 1. 36 & 2.27 & & 28.55 \\
\hline $1905-6$. & & & .02 & & 3.00 & 2.04 & 11.66 & 5. 76 & 9.82 & 1.83 & 3.15 & 1.15 & 38.43 \\
\hline $1906-7$. & & Tr. & .28 & .05 & 1. 92 & 10.31 & 9.74 & 4. 76 & 13.80 & 1.14 & .42 & .92 & 43. 34 \\
\hline 1907 & $\mathrm{Tr}$. & & .01 & 1.62 & .18 & 7.77 & 5.02 & 4.26 & 1.95 & .70 & 2.39 & .02 & 23.92 \\
\hline & 0 & 0 & & & 2.63 & 2.96 & 18.18 & 9.49 & 4.05 & & .11 & & 37.42 \\
\hline 1909 & & 0 & & 1.77 & 2.59 & 6.87 & 7.29 & 3.12 & 3.28 & .91 & .12 & .07 & 26.02 \\
\hline $1910-11$. & .04 & 0 & .25 & 1.06 & .94 & 1.77 & 15.76 & 4. 37 & 7.00 & 1.35 & .75 & 0 & 33.29 \\
\hline 1911-12. & & & & .46 & 1.21 & 3. 22 & 4.44 & .50 & 3.96 & 2.70 & 1.31 & .44 & 18. 24 \\
\hline $1912-13 \ldots$ & & & 2.01 & & 2.34 & 2.28 & 5.42 & .48 & 3.40 & .94 & & .07 & 17.88 \\
\hline $1913-14 \ldots$ & .06 & .10 & & & 5.34 & 6.05 & 11.57 & 5.24 & 2.31 & 2.01 & 1.80 & 1.13 & 35.61 \\
\hline Mean.. & .01 & .02 & .39 & 1.55 & 2.87 & 5.96 & 6.05 & 4. 60 & 5.42 & 2.63 & 1.53 & 0.39 & 31.42 \\
\hline
\end{tabular}

$a$ Records of J. S. Weather Bureau. 
138 contributions to Hydrology of united states, 1914.

Records of precipitation in or near the Alameda Creek drainage basin (in inches)-Contd.

Livermore, Alameda County.a

[Elevation 485 feet.]

\begin{tabular}{|c|c|c|c|c|c|c|c|c|c|c|c|c|c|}
\hline Season. & July. & Aug. & Sept. & Oct. & Nov. & Dec. & Jan. & Feb. & Mar. & Apr. & May. & June. & $\begin{array}{c}\text { Total } \\
\text { for } \\
\text { season. }\end{array}$ \\
\hline 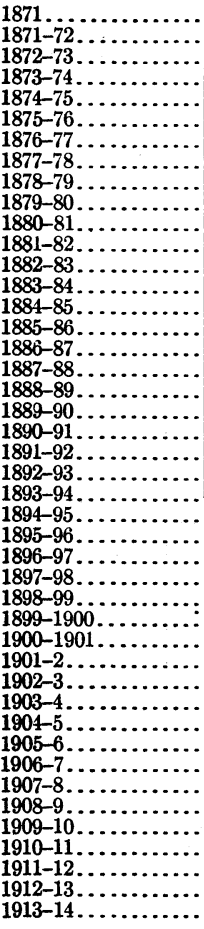 & $\begin{array}{l}0 \\
0 \\
0 \\
0 \\
0 \\
0 \\
0 \\
0 \\
0 \\
0 \\
0 \\
0 \\
0 \\
0 \\
0 \\
0.40 \\
0 \\
0 \\
0 \\
0 \\
0 \\
0 \\
0 \\
0 \\
0 \\
0 \\
0 \\
0 \\
0 \\
0 \\
0 \\
\operatorname{Tr} . \\
0 \\
\operatorname{Tr} . \\
0 \\
\mathrm{Tr} . \\
0 \\
\mathrm{Tr} . \\
0 \\
\mathrm{Tr} . \\
\mathrm{Tr} . \\
\mathrm{Tr} . \\
.27\end{array}$ & $\begin{array}{l}0 \\
\text { Tr. } \\
0 \\
0 \\
0 \\
0 \\
0 \\
0 \\
0 \\
0 \\
0 \\
0 \\
0 \\
.10 \\
0 \\
0 \\
0 \\
0 \\
0 \\
0 \\
0 \\
0 \\
0 \\
0 \\
0 \\
.73 \\
0 \\
0 \\
0 \\
0 \\
0 \\
.13 \\
0 \\
.32 \\
0 \\
0 \\
0 \\
0 \\
0 \\
0 \\
0 \\
\cdots .02\end{array}$ & $\begin{array}{l}0 \\
0 \\
0 \\
.30 \\
0 \\
0 \\
0 \\
0 \\
0 \\
0 \\
\mathrm{Tr} . \\
.34 \\
.35 \\
.30 \\
.05 \\
0 \\
.80 \\
.76 \\
0 \\
1.20 \\
1.32 \\
.45 \\
0 \\
1.45 \\
.22 \\
.55 \\
.06 \\
.95 \\
0 \\
.18 \\
.68 \\
0 \\
0 \\
1.62 \\
\mathrm{Tr} . \\
.20 \\
\mathrm{Tr} . \\
.03 \\
.62 \\
.10 \\
\mathrm{Tr} . \\
.48 \\
\mathrm{Tr} .\end{array}$ & $\begin{array}{l}\text { Tr. } \\
0 \\
.42 \\
1.67 \\
0 \\
1.26 \\
1.27 \\
.24 \\
.83 \\
0 \\
.08 \\
1.52 \\
1.52 \\
1.14 \\
0 \\
.30 \\
0 \\
0 \\
3.94 \\
0 \\
.05 \\
1.65 \\
0 \\
1.15 \\
.83 \\
1.48 \\
1.43 \\
.74 \\
2.52 \\
1.93 \\
.70 \\
.47 \\
\text { Tr. } \\
1.00 \\
0 \\
.03 \\
.81 \\
.27 \\
.75 \\
.29 \\
.43 \\
.71 \\
0\end{array}$ & $\begin{array}{r}1.13 \\
1.22 \\
.70 \\
2.03 \\
7.23 \\
.10 \\
1.29 \\
.31 \\
1.06 \\
.65 \\
.78 \\
1.48 \\
.57 \\
.02 \\
6.20 \\
.70 \\
.61 \\
3.80 \\
2.95 \\
0 \\
.38 \\
4.97 \\
1.59 \\
.50 \\
1.69 \\
3.02 \\
.52 \\
.25 \\
2.49 \\
4.48 \\
1.90 \\
2.07 \\
2.16 \\
.78 \\
1.61 \\
1.34 \\
.04 \\
.60 \\
1.68 \\
.10 \\
.29 \\
\because .79 \\
2.47\end{array}$ & $\begin{array}{l}1 . .69 \\
3.87 \\
4.48 \\
.20 \\
1.62 \\
0 \\
.73 \\
.17 \\
1.94 \\
7.75 \\
1.97 \\
.38 \\
.44 \\
6.22 \\
1.94 \\
.81 \\
3.51 \\
2.21 \\
8.63 \\
3.31 \\
4.42 \\
7.27 \\
2.14 \\
8.56 \\
1.28 \\
1.71 \\
1.31 \\
1.61 \\
2.07 \\
1.06 \\
.74 \\
.87 \\
.59 \\
1.42 \\
1.18 \\
6.45 \\
3.90 \\
1.55 \\
5.77 \\
1.32 \\
1.71 \\
.81 \\
3.17\end{array}$ & $\begin{array}{r}1.42 \\
2.15 \\
1.04 \\
2.96 \\
5.40 \\
2.68 \\
2.47 \\
4.61 \\
2.83 \\
1.48 \\
2.40 \\
1.07 \\
2.38 \\
4.03 \\
1.72 \\
4.20 \\
.90 \\
3.20 \\
.46 \\
5.24 \\
.54 \\
.84 \\
3.02 \\
4.97 \\
6.83 \\
7.16 \\
1.89 \\
1.47 \\
2.60 \\
2.44 \\
2.69 \\
b 3.99 \\
3.19 \\
.89 \\
2.43 \\
15.56 \\
3.07 \\
2.27 \\
10.18 \\
2.50 \\
12.60 \\
2.66 \\
\hdashline 7.10 \\
\hdashline .10\end{array}$ & $\begin{array}{r}1.93 \\
2.69 \\
3.73 \\
1.03 \\
1.20 \\
3.01 \\
.56 \\
6.73 \\
1.78 \\
1.80 \\
2.62 \\
1.72 \\
.63 \\
5.29 \\
.36 \\
.24 \\
6.23 \\
.94 \\
.67 \\
3.71 \\
4.18 \\
1.08 \\
3.12 \\
5.36 \\
1.56 \\
.17 \\
3.54 \\
1.78 \\
.08 \\
.34 \\
5.15 \\
3.52 \\
.94 \\
4.18 \\
2.30 \\
2.67 \\
1.86 \\
1.35 \\
3.96 \\
1.14 \\
1.42 \\
.20 \\
.38 \\
2.11\end{array}$ & $\begin{array}{r}0.36 \\
.65 \\
.68 \\
1.34 \\
.35 \\
4.39 \\
1.10 \\
2.01 \\
2.49 \\
1.45 \\
1.06 \\
4.85 \\
3.45 \\
5.92 \\
.78 \\
1.18 \\
.23 \\
2.51 \\
5.15 \\
2.85 \\
2.50 \\
3.96 \\
3.68 \\
.81 \\
1.81 \\
1.50 \\
4.01 \\
.78 \\
4.81 \\
1.11 \\
.95 \\
2.69 \\
5.65 \\
3.71 \\
3.12 \\
5.18 \\
8.85 \\
.75 \\
1.94 \\
1.90 \\
4.45 \\
1.99 \\
1.65 \\
.66\end{array}$ & $\begin{array}{r}1.25 \\
.43 \\
.15 \\
.95 \\
0.73 \\
.73 \\
.13 \\
.96 \\
.75 \\
6.51 \\
1.93 \\
1.03 \\
1.50 \\
2.70 \\
1.29 \\
2.36 \\
1.60 \\
.60 \\
.51 \\
.86 \\
1.88 \\
.90 \\
1.40 \\
.58 \\
1.26 \\
3.11 \\
.24 \\
.45 \\
.35 \\
.86 \\
1.80 \\
.75 \\
.81 \\
1.56 \\
.93 \\
.95 \\
.47 \\
.28 \\
0 \\
.10 \\
.69 \\
.73 \\
.54 \\
.76\end{array}$ & $\begin{array}{l}0.02 \\
0 \\
0 \\
.32 \\
.33 \\
.33 \\
.06 \\
1.34 \\
.91 \\
0 \\
.20 \\
2.18 \\
.20 \\
.08 \\
0 \\
0 \\
.66 \\
2.25 \\
.48 \\
.40 \\
1.30 \\
.73 \\
1.91 \\
1.25 \\
.39 \\
0 \\
.96 \\
.15 \\
1.10 \\
1.58 \\
.32 \\
.12 \\
.24 \\
1.89 \\
1.61 \\
.16 \\
.53 \\
\text { Tr. } \\
\text { Tr. } \\
.24 \\
.94 \\
.58 \\
.45\end{array}$ & 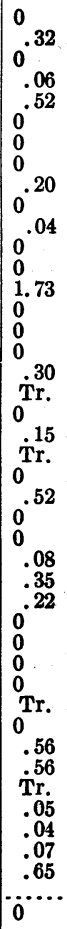 & $\begin{array}{r}19.06 \\
10.69 \\
12.26 \\
11.67 \\
19.99 \\
6.01 \\
17.66 \\
10.11 \\
15.98 \\
16.45 \\
11.70 \\
13.86 \\
22.75 \\
12.01 \\
16.17 \\
11.17 \\
13.13 \\
15.81 \\
28.66 \\
14.16 \\
14.25 \\
26.29 \\
17.16 \\
24.37 \\
16.35 \\
17.28 \\
9.11 \\
11.54 \\
12.93 \\
19.82 \\
12.48 \\
14.25 \\
13.33 \\
15.81 \\
19.32 \\
22.99 \\
9.93 \\
18.58 \\
14.50 \\
21.28 \\
9.60 \\
5.15 \\
17.01\end{array}$ \\
\hline Mean & .02 & .00 & & .73 & 1.61 & 2.86 & 3.34 & 2.26 & 2.53 & 1.08 & & .15 & 15.49 \\
\hline
\end{tabular}


Records of precipitation in or near the Alameda Creek drainage basin (in inches)-Contd

\section{Niles, Alameda County. $a$}

[Elevation 87 feet.]

\begin{tabular}{|c|c|c|c|c|c|c|c|c|c|c|c|c|c|}
\hline Season. & July. & Aug. & Sept. & Oct. & Nov. & Dec. & Jan. & Feb. & Mar. & Apr. & May. & June. & $\begin{array}{c}\text { Total } \\
\text { for } \\
\text { season. }\end{array}$ \\
\hline 1870 & & & & & & 2.04 & 2.00 & 2.12 & 0.27 & 0.82 & 0 & 0 & \\
\hline 72 & 0 & 0 & 0 & 0.05 & $1 . \overline{56}$ & 11.91 & 2.15 & 4.32 & 1.30 & .94 & 0 & 42 & 22.65 \\
\hline 73. & 0 & $\mathrm{Tr}$ & 0 & .11 & 2.00 & 5.00 & 1.07 & 5.15 & .66 & .32 & 0 & & 14.31 \\
\hline $4 \ldots$ & 0 & & 0 & .66 & .55 & 4.00 & 3.53 & $\begin{array}{r}.82 \\
\end{array}$ & 3.14 & 1.40 & 0 & & 14.10 \\
\hline$-75 .$. & 0 & 0 & .07 & 2.44 & 4.33 & & 3.44 & .23 & .69 & & .13 & & 11.81 \\
\hline-76. & 0 & 0 & & .10 & 8.83 & 3.52 & 4.36 & 4. 12 & 3.40 & .85 & .70 & 0 & 25.88 \\
\hline$-77 \ldots$ & 0 & 0 & 0 & 2.75 & .15 & 0 & 3.03 & .67 & 1.02 & .42 & 1.03 & 0 & 9.34 \\
\hline$-78 \ldots$ & 0 & 0 & 0 & .23 & 1.16 & 1.96 & 7.67 & 8.50 & 3.58 & 1.50 & .07 & 0 & 24.67 \\
\hline 79. & 0 & 0 & 0 & & .43 & .34 & 3.10 & 2.66 & 3.89 & 2.09 & 1. 24 & & 14.54 \\
\hline$-80 \ldots$ & 0 & 0 & 0 & & 1.57 & 3.41 & 94 & 1.29 & 1.60 & 5.87 & 1.35 & & 17.70 \\
\hline $1880-81$ & 0 & 0 & 0 & & .63 & 9.03 & 4.36 & 3.08 & 1.06 & 1.53 & & .37 & 20.06 \\
\hline-82. & 0 & 0 & .08 & .38 & .87 & 2.59 & 1.52 & 1.74 & 4.75 & 1.17 & .45 & Tr. & 13.55 \\
\hline $82-83$. & 0 & 0 & .44 & 1.29 & 2.30 & .81 & 1.44 & .30 & 2.77 & 1.46 & 2.99 & & 13.80 \\
\hline$-84 \ldots$ & 0 & 0 & 71 & 1.47 & .88 & 1. 21 & 3.78 & 6.18 & 5.41 & 3.74 & .18 & 2.69 & 26.25 \\
\hline$-85 .$. & 0 . & 0 & 34 & 1.30 & 0 & 5.75 & 1.58 & .15 & .66 & .92 & 0 & 0 & 10.60 \\
\hline 86 & $0^{\circ}$ & 0 & & .35 & 8.78 & 1.92 & 5.63 & .73 & 1. 67 & 4.19 & .25 & .01 & 23. 53 \\
\hline$\ldots .$. & Tr. & 0 & & 64 & 1.29 & 1.14 & 1.07 & 1.01 & 1.87 & .14 & 14 & .07 & 14.85 \\
\hline$-88 \ldots \ldots \ldots \ldots$ & .01 & 0 & .51 & Tr. & .84 & 3.25 & 3.99 & 1.80 & 3.07 & 15 & 78 & .40 & 14.88 \\
\hline-89. & 0 & $\mathrm{Tr}$ & .39 & .07 & 3.87 & 3.53 & .55 & .42 & 5.59 & .95 & 1.5 & .01 & \\
\hline 18 & 0 & 0 & & 4.30 & 3.44 & 12.13 & 7.18 & 3.63 & 3.03 & 1.1 & 1.0 & & 35.91 \\
\hline-91 . & 0 & 0 & .45 & 0 & 0 & 3.05 & .6 & 4.72 & 2.57 & 2.2 & 1.10 & .06 & 14.83 \\
\hline 92. & 0 & 0 & .21 & .06 & .37 & 6.45 & 1.12 & 1.41 & 3.71 & 1.10 & 1.96 & Tr. & 16. 39 \\
\hline 189 & .03 & 0 & .01 & 1.53 & 3.49 & 5.28 & 2.65 & 2.73 & 5.58 & 1.67 & .47 & .02 & 23.46 \\
\hline 189 & & 0 & .14 & & 2.44 & 2.69 & 7.28 & 4.99 & 1.23 & .54 & 1.94 & & 21.91 \\
\hline $1894-95$ & Tr. & 0 & 1.42 & 1.49 & .84 & 9.45 & 6.81 & 2.55 & 2.15 & 1. 73 & .86 & & 27.30 \\
\hline ..... & Tr. & 0 & .75 & .72 & 1.80 & 1. 36 & 7.45 & .30 & 1.66 & 4.47 & 1.07 & 0 & 19.58 \\
\hline ..... & .10 & & .57 & 1.22 & 6.16 & 2.91 & 2. & 4.47 & 4.98 & .56 & & & \\
\hline 18 & & & Tr. & 2.42 & .92 & 1.9 & 1.7 & 2.20 & .88 & .3 & 1.2 & 2 & 11.99 \\
\hline 18 & & & 1. 46 & 1.33 & .68 & 1.65 & 2.78 & .49 & 6.09 & 26 & .66 & 4 & 15.89 \\
\hline 18 & Tr. & & & 4.24 & 3. 30 & 2.91 & 3.49 & .87 & 1.87 & .91 & & & 18.65 \\
\hline 190 & Tr. & & .02 & 2.02 & 4.67 & 1.65 & 3.31 & 7.87 & 1.02 & 3.05 & 1. 26 & & 87 \\
\hline 190 & Tr. & & & & 1.78 & 1.19 & 1.48 & 5.80 & 3. 27 & 1.31 & 1. 40 & 0 & .47 \\
\hline 19 & 0 & & & 1.06 & 2.49 & 1.46 & 4.2 & 1.36 & 5.82 & .75 & 0 & 0 & \\
\hline-4 & 0 & Tr. & 0 & .13 & 3.52 & & 1.3 & 5.56 & 4. 70 & 2.11 & .3 & .08 & \\
\hline 19 & 0 & .48 & 2.80 & 2.25 & 1.45 & 2.1 & 3. & 3.21 & 3.51 & 1.40 & 2.8 & $\mathrm{Tr}$ & \\
\hline & 0 & Tr. & .02 & Tr. & 2.36 & 2.1 & 5.2 & 4.48 & 5.80 & 1.33 & 1.5 & .8 & \\
\hline & 0 & Tr. & .31 & .10 & 1.97 & 6.8 & 4. 74 & 3.57 & 10.09 & .77 & 0 & & 28.35 \\
\hline & 0 & & & 1. 40 & .03 & 4.42 & 2.97 & 2.57 & .80 & .23 & .48 & 0 & 12.90 \\
\hline & 0 & & 0 & .55 & 1.18 & 1. 66 & 9.00 & 7. 06 & 3. 19 & & 0 & 0 & 64 \\
\hline & 0 & 0 & .66 & 1. 08 & 1.86 & 5.01 & 4. 44 & 2.26 & 2.55 & .47 & 0 & 0 & 18.33 \\
\hline & 0 & 0 & 0 & & 14 & .91 & 13.31 & 4.93 & .85 & .44 & 0 & & \\
\hline & 0 & 0 & 0 & 1. 11 & & 2.47 & & .20 & 2.75 & 1.84 & .71 & & 12. \\
\hline 191 & & & 0 & .28 & 1.04 & 1.5 & 3. & .31 & 1.68 & .66 & .7 & & 9.6 \\
\hline & .08 & .05 & 0 & .02 & 2.97 & 4. 19 & 6. 76 & 2.94 & .68 & .69 & .21 & .32 & 18.91 \\
\hline Mean & Tr. & .03 & .28 & .97 & 2.08 & 3.36 & 3.75 & 3.00 & 2.91 & 1.37 & .73 & .19 & 18.61 \\
\hline
\end{tabular}

a 1870-71 to 1911-12, records of U.S. Weather Bureau; 1912-13 and 1913-14, records of Southern Pacific Co.

Pleasanton, Alameda County. $a$

[Elevation 355 feet.]

\begin{tabular}{|c|c|c|c|c|c|c|c|c|c|c|c|c|c|}
\hline Season. & July. & Aug. & Sept. & Oct. & Nov. & Dec. & Jan. & Feb. & Mar. & Apr. & May. & June. & $\begin{array}{c}\text { Total } \\
\text { for } \\
\text { season. }\end{array}$ \\
\hline 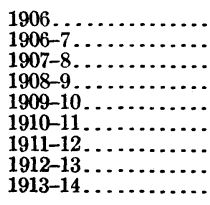 & $\begin{array}{l}0 \\
0 \\
0 \\
0 \\
0 \\
0 \\
0 \\
.12\end{array}$ & $\begin{array}{l}0 \\
0 \\
0 \\
0 \\
0 \\
0 \\
0 \\
0\end{array}$ & $\begin{array}{l}\dddot{3} \\
0 \\
.25 \\
.65 \\
0 \\
0 \\
0 \\
0\end{array}$ & $\begin{array}{l}70 \\
1.17 \\
.50 \\
.29 \\
0 \\
0 \\
0.30\end{array}$ & $\begin{array}{r}1.90 \\
1.25 \\
1.63 \\
.60 \\
.28 \\
.67 \\
3.73\end{array}$ & $\begin{array}{l}7.57 \\
4.20 \\
1.26 \\
4.68 \\
1.41 \\
2.04 \\
1.32 \\
2.89\end{array}$ & $\begin{array}{r}3.94 \\
2.80 \\
10.73 \\
4.44 \\
12.60 \\
1.54 \\
4.60 \\
14.94\end{array}$ & $\begin{array}{r}1.29 \\
1.15 \\
4.51 \\
1.75 \\
.20 \\
.25 \\
2.18\end{array}$ & $\begin{array}{r}8.42 \\
1.25 \\
2.03 \\
2.29 \\
2.83 \\
2.56 \\
2.78 \\
.47\end{array}$ & $\begin{array}{r}0.20 \\
.42 \\
.20 \\
0 \\
.33 \\
.80 \\
1.27 \\
.27 \\
.83\end{array}$ & $\begin{array}{r}1.59 \\
.07 \\
.30 \\
. .48 \\
0 \\
.13 \\
.81 \\
.48 \\
1.9\end{array}$ & $\begin{array}{l}0.57 \\
.20 \\
0 \\
0 \\
0 \\
0 \\
.87 \\
0.19\end{array}$ & $\begin{array}{r}23.81 \\
11.07 \\
b 21.11 \\
14.96 \\
19.98 \\
9.57 \\
10.67 \\
17.05\end{array}$ \\
\hline Mean.............. & .01 & 0 & .11 & .16 & 1.27 & 3.17 & 6.95 & 1.50 & 2.83 & .39 & .46 & .20 & 16.03 \\
\hline
\end{tabular}




\section{CONTRIBUtIONS TO HYDROLOGY OF UNITED STATES, 1914.}

Records of precipitation in or near the Alameda Creek drainage basin (in inches)-Contd.

\section{San Jose, Santa Clara County.a}

[Elevation 95 feet.]

\begin{tabular}{|c|c|c|c|c|c|c|c|c|c|c|c|c|c|}
\hline Season. & July. & Aug. & Sept. & Oct. & Nov. & Dec. & Jan. & Feb. & Mar. & Apr. & May. & June. & $\begin{array}{c}\text { Total } \\
\text { for } \\
\text { season. }\end{array}$ \\
\hline 874. & & & & & & & 2.61 & 0.77 & 2.83 & 0.87 & 0.21 & & \\
\hline & 0 & 0 & 0.10 & 1.81 & 1.91 & 0.08 & 2.75 & .41 & .39 & Tr. & $\mathrm{Tr}$. & & 7.90 \\
\hline $1875-76$ & 0 & 0 & & Tr. & 6.10 & 2.12 & 4. 08 & 3.41 & 3.11 & .41 & .25 & & 19.47 \\
\hline $1876-77$. & Tr. & 0 & .11 & 1.17 & .01 & & 2.23 & .48 & .78 & & .05 & 0 & 4.83 \\
\hline $1877-78$ & 0 & 0 & 0 & .37 & .85 & 1.87 & 5.53 & 6.94 & 2.22 & 1.48 & .02 & 0 & 19.28 \\
\hline 1878-79. & 0 & 0 & .48 & .80 & .76 & .97 & 1.48 & 3.18 & 5.85 & 1.24 & 1.58 & .06 & 16.40 \\
\hline 1879-80. & 0 & 0 & 0 & .87 & 1.79 & 2.99 & 1.48 & 1.35 & .96 & 3.66 & .67 & & 13. 77 \\
\hline $80-81$. & 0 & 0 & 0 & 0 & .49 & 5.60 & 2.12 & 2.04 & .80 & 1.28 & & .12 & 12.45 \\
\hline-82 & 0 & 0 & .02 & .45 & .88 & 1.83 & 1.17 & 1.49 & 4.26 & 1.10 & .55 & & 11.75 \\
\hline 1882-83. & 0 & 0 & .04 & .87 & 1.32 & .82 & 1.06 & .94 & 2.70 & .66 & 2.18 & 0 & 10.59 \\
\hline 1883-84. & 0 & 0 & .09 & .67 & .28 & .37 & 3.18 & 3.68 & 6.23 & 3.38 & .05 & 2.15 & 20.08 \\
\hline 1884-85. & 0 & 0 & .08 & 1.50 & .06 & 3.90 & 1.83 & .18 & .86 & 2.75 & .11 & Tr. & 11.27 \\
\hline 1885-86. & 0 & 0 & 0 & .06 & 7.39 & 2.11 & 3.59 & 1.12 & 1.89 & 4. 47 & & 0 & 20.63 \\
\hline $1886-87$ & .03 & 0 & 0 & .49 & .73 & .71 & .68 & 6.81 & .63 & 1. 28 & 0 & 0 & 11.36 \\
\hline $1887-88$. & .02 & 0 & .61 & .03 & .70 & 2.53 & 3.06 & 1.09 & 3.00 & .31 & .60 & .22 & 12.17 \\
\hline 1888-89. & & 0 & .60 & 0 & 3.88 & 2.44 & .50 & .70 & 5.80 & .79 & .96 & & 15. 71 \\
\hline 1889-90. & 0 & 0 & 0 & 4. 48 . & 1.73 & 10.55 & 6.52 & 3.64 & 2.08 & .55 & .75 & & 30.30 \\
\hline & 0 & 0 & .05 & & .05 & 2.40 & .55 & 5. 27 & 2.47 & 1. 79 & .26 & .05 & 12. 88 \\
\hline 1891-92. & 0 & 0 & .37 & .08 & .46 & 5.84 & 1.11 & 1.60 & 4.75 & .65 & 1.60 & .05 & 16. 51 \\
\hline $1892-93$ & 0 & 0 & 0 & 1.00 & 4.00 & 7.77 & 2.95 & 2.68 & 5.12 & 1.35 & .30 & & 25.17 \\
\hline & 0 & 0 & 0 & & .81 & 1.69 & 4.73 & 2.61 & .69 & .63 & 1.36 & .40 & 12.92 \\
\hline 1894-95. & 0 & 0 & 1.08 & 1.32 & .55 & 7.80 & 6.28 & 1.42 & 1.46 & 2.05 & 1.36 & & 23.32 \\
\hline-96. & 0 & 0 & .05 & .83 & 1.08 & .84 & 5.17 & .27 & 2.22 & 2.79 & .44 & 0 & 13. 69 \\
\hline & .01 & .74 & .32 & 1.03 & 2.82 & 2.55 & 1.68 & 3.43 & 2.64 & .91 & .16 & Tr. & 16.56 \\
\hline $1897-98$ & 0 & 0 & .21 & 1.01 & .37 & 1.20 & .93 & 1.93 & .52 & .20 & .44 & .06 & 6.87 \\
\hline 1898-99. & 0 & 0 & 1.13 & .61 & .45 & .44 & 1.88 & .21 & 4.17 & .48 & .65 & Tr. & 10.02 \\
\hline $1899-1900$ & & 0 & & 3. 26 & 2.70 & 1.43 & 2.05 & .4 & 1.36 & $1 . t$ & .96 & .01 & 13. 87 \\
\hline $0-190$ & .02 & 0 & .17 & .62 & 4.36 & 1.32 & 3.98 & 5.47 & .75 & 2.37 & .82 & Tr. & 19. 88 \\
\hline 1901-2. & 0 & Tr. & .44 & 1.00 & 1.06 & .43 & .81 & 4.42 & 2.65 & 1.29 & .88 & & 12.98 \\
\hline $1902-3$. & 0 & 0 & 0 & .95 & 2.18 & .92 & 2.74 & 1.27 & 4.99 & 84 & 0 & 0 & 13. 89 \\
\hline & 0 & 0 & 0 & .12 & .99 & .34 & 1.28 & 3. & 2.73 & 1.74 & .26 & 0 & 10.47 \\
\hline $1904-5$ & 0 & .25 & 1.94 & 1.43 & 1.20 & 2.28 & 2.70 & 2.6 & 2.73 & 1.01 & 1.77 & & 17.96 \\
\hline 19 & 0 & 0 & Tr. & 0 & 2.17 & 1.2 & 2.86 & 2. & 4.47 & .90 & .75 & .43 & 15.12 \\
\hline & 0 & 0 & .13 & .01 & .98 & 6. 39 & 4.61 & 1.8 & 7.75 & .46 & .08 & .42 & 22.71 \\
\hline $1907-8$ & Tr. & 0 & .06 & .98 & .13 & 3. 65 & 2.63 & 2.46 & 1.14 & .23 & .67 & .01 & 11. 69 \\
\hline 1908-9. & & & .09 & .19 & 1.11 & 1.54 & 7.69 & 4.87 & 2.77 & 0 & 0 & .05 & 18.31 \\
\hline & 0 & 0 & .75 & .72 & 1.27 & 5.37 & 2.31 & .8 & 2.84 & .41 & Tr. & .02 & 14.52 \\
\hline $1910-11$ & Tr. & 0 & .09 & .20 & .28 & .68 & 12.38 & 2.03 & 6.26 & .45 & 1.21 & .07 & 22.65 \\
\hline $1911-12$ & 0 & & 0 & .80 & .18 & 2.03 & 1.36 & .30 & 2.80 & 1.95 & .70 & .46 & 10.58 \\
\hline & Tr. & 0 & .71 & .21 & .29 & .43 & 2.29 & $: 09$ & 1.17 & .38 & .77 & .01 & 6.35 \\
\hline $1913-14$ & .09 & .08 & Tr. & .02 & 4.10 & 3.00 & 6.23 & 3.94 & .90 & .65 & .19 & .25 & 19.45 \\
\hline Mean. & Tr. & .03 & .24 & .76 & 1.56 & 2.51 & 3.05 & 2. 28 & 2.77 & .121 & .55 & .13 & 15. 09 \\
\hline
\end{tabular}

$a$ Records of U. S. Weather Bureau. 
Records of precipitation in or near the Alameda Creek drainage basin (in inches)-Contd.

Sunol, Alameda County. $a$

[Elevation 227 feet.]

\begin{tabular}{|c|c|c|c|c|c|c|c|c|c|c|c|c|c|}
\hline Season. & July. & Aug. & Sept. & Oct. & Nov. & Dec. & Jan. & Feb. & Mar. & Apr. & May. & June. & $\begin{array}{c}\text { Total } \\
\text { for } \\
\text { season. }\end{array}$ \\
\hline $\begin{array}{l}1889 \ldots \ldots \ldots \\
1889-90 \ldots \ldots \ldots \\
1890-91 \ldots \ldots \ldots \\
1891-92 \ldots \ldots \ldots \\
1892-93 \ldots \ldots \ldots \\
1893-94 \ldots \ldots \ldots \\
1894-95 \ldots \ldots \ldots \\
1895-96 \ldots \ldots \ldots \\
1896-97 \ldots \ldots \ldots \\
1897-98 \ldots \ldots \ldots \\
1898-99 \ldots \ldots \ldots\end{array}$ & $\begin{array}{l}0 \\
0 \\
0 \\
0 \\
0 \\
0 \\
0 \\
0 \\
.20 \\
0 \\
0\end{array}$ & $\begin{array}{l}0 \\
0 \\
0 \\
0 \\
0 \\
0 \\
0 \\
0 \\
0_{0}^{-30} \\
0\end{array}$ & $\begin{array}{l}0 \\
.90 \\
1.00 \\
0 \\
.07 \\
1.45 \\
.37 \\
.46 \\
0.80\end{array}$ & $\begin{array}{l}4.69 \\
0 \\
.12 \\
1.80 \\
.18 \\
1.39 \\
.45 \\
1.33 \\
1.68 \\
1.11\end{array}$ & $\begin{array}{r}3.69 \\
.14 \\
.37 \\
6.35 \\
2.09 \\
.60 \\
2.00 \\
4.31 \\
.48 \\
.52\end{array}$ & $\begin{array}{r}11.36 \\
3.51 \\
6.27 \\
4.82 \\
2.65 \\
9.93 \\
1.17 \\
2.61 \\
1.89 \\
1.98\end{array}$ & $\begin{array}{l}0.67 \\
8.36 \\
3.78 \\
.77 \\
2.67 \\
6.64 \\
6.87 \\
7.17 \\
2.17 \\
1.78 \\
3.19\end{array}$ & $\begin{array}{r}0.35 \\
4.98 \\
1.06 \\
1.21 \\
2.79 \\
6.38 \\
2.73 \\
.32 \\
4.32 \\
2.38 \\
.31\end{array}$ & $\begin{array}{r}5.52 \\
3.63 \\
1.89 \\
3.87 \\
6.43 \\
1.32 \\
1.70 \\
1.10 \\
5.75 \\
.75 \\
6.72\end{array}$ & $\begin{array}{l}0.80 \\
1.21 \\
2.23 \\
1.12 \\
2.09 \\
.73 \\
1.66 \\
4.05 \\
.42 \\
.30 \\
.99\end{array}$ & $\begin{array}{r}2.75 \\
1.07 \\
.54 \\
1.81 \\
.24 \\
1.36 \\
.69 \\
.88 \\
.35 \\
1.04 \\
.56\end{array}$ & $\begin{array}{l}0 \\
0 \\
0 \\
0 \\
0 \\
.30 \\
0 \\
.08 \\
.08 \\
.11 \\
.11\end{array}$ & $\begin{array}{l}38.99 \\
14.05 \\
16.54 \\
27.19 \\
21.72 \\
27.02 \\
17.59 \\
22.22 \\
10.51 \\
16.29\end{array}$ \\
\hline $\begin{array}{c}\text { Mean, } 1889 \text { to } 1898- \\
99\end{array}$ & .02 & .03 & .51 & 1.28 & 2.06 & 4.62 & 4.01 & 2.44 & 3.52 & 1. 42 & 1.03 & .06 & 21.00 \\
\hline 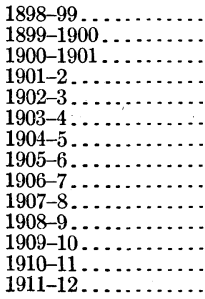 & $\begin{array}{l}0 \\
0 \\
0 \\
0 \\
0 \\
0 \\
0 \\
0 \\
0 \\
0 \\
0 \\
0 \\
0 \\
0\end{array}$ & $\begin{array}{l}0 \\
0 \\
0 \\
0 \\
0 \\
0 \\
0 \\
0 \\
0 \\
0 \\
0 \\
0 \\
0 \\
0\end{array}$ & $\begin{array}{l}0^{.83} \\
0 \\
0^{.93} \\
0 \\
2.57 \\
0 \\
0^{.25} \\
.48 \\
.71 \\
0 \\
0\end{array}$ & $\begin{array}{l}.86 \\
3.90 \\
2.58 \\
1.31 \\
.76 \\
.06 \\
1.96 \\
0 \\
0 \\
1.43 \\
.38 \\
.89 \\
.68 \\
.90\end{array}$ & $\begin{array}{l}.83 \\
4.30 \\
5.21 \\
1.92 \\
2.67 \\
4.73 \\
1.22 \\
2.42 \\
1.69 \\
0 \\
.84 \\
2.05 \\
.23 \\
1.10\end{array}$ & $\begin{array}{l}2.10 \\
4.30 \\
1.81 \\
2.10 \\
1.30 \\
.72 \\
2.28 \\
2.47 \\
7.44 \\
5.81 \\
2.39 \\
3.54 \\
1.38 \\
3.25\end{array}$ & $\begin{array}{r}3.40 \\
3.29 \\
2.97 \\
1.27 \\
4.88 \\
2.17 \\
2.70 \\
6.83 \\
4.44 \\
3.53 \\
11.72 \\
4.97 \\
14.83 \\
2.90\end{array}$ & $\begin{array}{l}.30 \\
1.33 \\
7.61 \\
5.84 \\
1.51 \\
6.04 \\
3.48 \\
3.74 \\
3.18 \\
2.76 \\
6.03 \\
2.34 \\
3.26 \\
.41\end{array}$ & $\begin{array}{r}9.05 \\
2.10 \\
1.51 \\
4.23 \\
5.95 \\
5.88 \\
4.88 \\
6.91 \\
11.03 \\
1.32 \\
3.30 \\
3.72 \\
6.38 \\
3.23\end{array}$ & $\begin{array}{r}2.00 \\
2.12 \\
2.57 \\
1.28 \\
1.95 \\
2.20 \\
1.38 \\
1.65 \\
.55 \\
.31 \\
0.49 \\
.49 \\
1.41 \\
1.90\end{array}$ & $\begin{array}{r}.83 \\
.76 \\
1.11 \\
.66 \\
0.36 \\
.36 \\
.50 \\
.52 \\
.70 \\
1.02 \\
0 \\
0 \\
.26 \\
1.24\end{array}$ & $\begin{array}{l}.21 \\
0 \\
0 \\
0 \\
0 \\
0 \\
0 \\
.67 \\
.50 \\
0 \\
0 \\
0 \\
0 \\
.88\end{array}$ & $\begin{array}{l}20.41 \\
22.27 \\
25.37 \\
19.54 \\
19.02 \\
22.16 \\
23.29 \\
25.21 \\
29.78 \\
16.18 \\
25.14 \\
18.53 \\
28.43 \\
15.81\end{array}$ \\
\hline $\begin{array}{c}\text { Mean, } 1898-99 \text { to } \\
1911-12 \ldots \ldots \ldots .\end{array}$ & 0 & .03 & .41 & 1.12 & 2.09 & 2.92 & 4.99 & 3.42 & 4.96 & 1.41 & .71 & .16 & 22.22 \\
\hline
\end{tabular}

$a$ i889 to 1898-99, records of Southern Pacific Co.; 1898-99 to 1911-12, records of Spring Valley Water Co. (The future water supply of San Francisco from the conservation and use of its present resources, p. 472, Oct. 31,1912 .)

$71520^{\circ}$ —WSP $345-15-10$ 
Monthly discharge, in acre-feet, of Alameda Creek, at Sunol dam, near Sunol, Cal.

[Compiled from records of Spring Valley Water Co. Drainage area 620.5 square miles.]

\begin{tabular}{|c|c|c|c|c|c|c|c|c|c|c|c|c|c|c|}
\hline & $1900-1901$ & $1901-2$ & $1902-3$ & $1903-4$ & $1904-5$ & $1905-6$ & $1906-7$ & $1907-8$ & $1908-9$ & 1909-10 & $1910-11$ & 1911-12 & 1912-13 & $1913-14$ \\
\hline 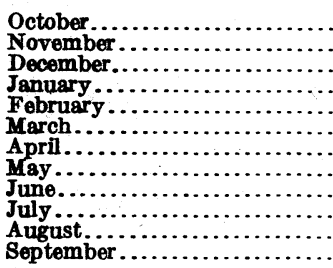 & $\begin{array}{r}602 \\
23,400 \\
3,200 \\
16,600 \\
55,100 \\
9,880 \\
3,920 \\
3,560 \\
1,380 \\
571 \\
156 \\
0\end{array}$ & $\begin{array}{r}0 \\
31 \\
1,070 \\
437 \\
29,000 \\
42,100 \\
7,580 \\
2,400 \\
862 \\
310 \\
141 \\
0\end{array}$ & $\begin{array}{r}323 \\
46 \\
16,400 \\
18,200 \\
39,800 \\
30,900 \\
2,390 \\
200 \\
59 \\
0 \\
0\end{array}$ & $\begin{array}{r}0 \\
1,410 \\
21 \\
358 \\
27,500 \\
49,900 \\
16,200 \\
5,540 \\
550 \\
75 \\
14 \\
2\end{array}$ & $\begin{array}{r}1 \\
1 \\
665 \\
3,240 \\
11,600 \\
21,500 \\
6,080 \\
4,880 \\
469 \\
7 \\
3 \\
0\end{array}$ & $\begin{array}{r}0 \\
1 \\
7 \\
55,200 \\
22,000 \\
86,400 \\
27,500 \\
7,990 \\
3,430 \\
537 \\
100 \\
51\end{array}$ & $\begin{array}{r}2 \\
2 \\
19,700 \\
69,700 \\
28,800 \\
171,000 \\
22,700 \\
6,590 \\
2,440 \\
1,780 \\
709 \\
110\end{array}$ & $\begin{array}{r}127 \\
455 \\
4,560 \\
14,100 \\
13,900 \\
10,700 \\
1,620 \\
801 \\
45 \\
40 \\
22 \\
43\end{array}$ & $\begin{array}{r}13 \\
27 \\
78 \\
111,000 \\
103,000 \\
21,700 \\
4,810 \\
808 \\
320 \\
285 \\
1 \\
14\end{array}$ & $\begin{array}{r}101 \\
87 \\
9,480 \\
34,000 \\
14,400 \\
16,900 \\
6,660 \\
1,800 \\
343 \\
224 \\
81 \\
42\end{array}$ & $\begin{array}{r}17 \\
17 \\
63 \\
94,300 \\
42,000 \\
126,000 \\
6,160 \\
1,830 \\
336 \\
268 \\
228 \\
133\end{array}$ & $\begin{array}{r}110 \\
165 \\
212 \\
4,160 \\
1,580 \\
8,210 \\
1,700 \\
311 \\
15 \\
583 \\
0 \\
0\end{array}$ & $\begin{array}{r}0 \\
0 \\
0 \\
5,420 \\
120 \\
897 \\
20 \\
0 \\
0 \\
0 \\
0 \\
0\end{array}$ & $\begin{array}{r}0 \\
0 \\
10,800 \\
114,000 \\
51,400 \\
8,030 \\
2,260 \\
199 \\
65 \\
12 \\
0 \\
0\end{array}$ \\
\hline The year.. & 118,400 & 83,900 & 108,300 & 101,600 & 48,400 & 203,200 & 323,500 & 46,400 & 242,000 & 84,100 & 271,400 & 16,500 & 6,500 & 187,000 \\
\hline
\end{tabular}

Monthly discharge over Brightside weir, in acre-feet.

[Compiled from records of Spring Valley Water Co.]

\begin{tabular}{|c|c|c|c|c|c|c|c|c|c|c|c|}
\hline- & $1903-4$ & $1904-5$ & $1905-6$ & $1906-7$ & $1907-8$ & $1908-9$ & $1909-10$ & $1910-11$ & 1911-12 & 1912-13 & $1913-14$ \\
\hline 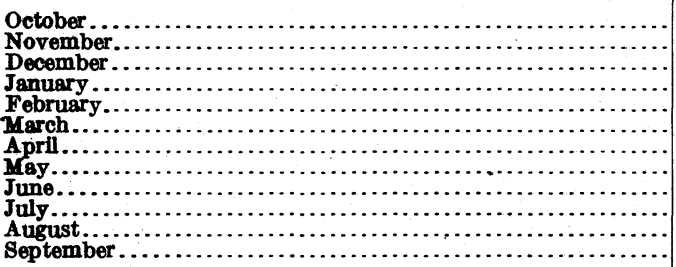 & $\begin{array}{l}1,021 \\
1,178 \\
1,548 \\
1,549 \\
1,385 \\
1,548 \\
1,490 \\
1,573 \\
1,526 \\
1,591 \\
1,442 \\
1,030\end{array}$ & $\begin{array}{l}1,118 \\
1,035 \\
1,243 \\
1,479 \\
1,340 \\
1,508 \\
1,493 \\
1,569 \\
1,544 \\
1,563 \\
1,385 \\
\mathbf{9 8 2}\end{array}$ & $\begin{array}{r}869 \\
824 \\
875 \\
1,108 \\
1,398 \\
1,534 \\
1,065 \\
648 \\
1,238 \\
1,423 \\
1,516 \\
1,493\end{array}$ & $\begin{array}{l}1,412 \\
1,301 \\
1,339 \\
1,446 \\
1,425 \\
1,151 \\
1,562 \\
1,668 \\
1,608 \\
1,647 \\
1,680 \\
1,575\end{array}$ & $\begin{array}{l}1,568 \\
1,520 \\
1,464 \\
1,402 \\
1,443 \\
1,593 \\
1,552 \\
1,609 \\
1,608 \\
1,599 \\
1,199 \\
1,060\end{array}$ & $\begin{array}{r}939 \\
903 \\
967 \\
1,022 \\
\mathbf{4 4 3} \\
\mathbf{1}, 215 \\
\mathbf{1}, 455 \\
\mathbf{1}, 628 \\
1,514 \\
1,493 \\
\mathbf{1}, 553 \\
\mathbf{1 , 4 7 4}\end{array}$ & $\begin{array}{l}1,531 \\
1,384 \\
1,460 \\
1,832 \\
1,555 \\
1,707 \\
1,666 \\
1,591 \\
1,475 \\
1,371 \\
1,518 \\
1,232\end{array}$ & $\begin{array}{r}684 \\
\mathbf{9 5 2} \\
\mathbf{1}, \mathbf{3 0 9} \\
\mathbf{1}, \mathbf{4 9 2} \\
\mathbf{1 , 0 7 4} \\
\mathbf{1 , 3 3 4} \\
\mathbf{1 , 4 8 2} \\
\mathbf{1 , 6 6 3} \\
\mathbf{1 , 5 8 7} \\
\mathbf{1 , 5 2 8} \\
\mathbf{1 , 6 7 0} \\
\mathbf{1 , 4 0 2}\end{array}$ & $\begin{array}{r}\mathbf{1}, 586 \\
\mathbf{1}, 510 \\
\mathbf{1}, \mathbf{5 1 5} \\
\mathbf{1}, \mathbf{5 7 7} \\
\mathbf{1 , 4 7 6} \\
\mathbf{1 , 6 1 0} \\
\mathbf{1 , 5 4 6} \\
\mathbf{1 , 6 0 2} \\
\mathbf{1 , 6 0 0} \\
\mathbf{1}, \mathbf{3 2 1} \\
\mathbf{1 , 1 2 0} \\
\mathbf{9 6 8}\end{array}$ & $\begin{array}{r}948 \\
769 \\
993 \\
1,295 \\
1,446 \\
1,658 \\
1,953 \\
1,697 \\
1,351 \\
1,120 \\
806 \\
1,001\end{array}$ & $\begin{array}{r}941 \\
971 \\
1,240 \\
2,024 \\
1,971 \\
2,185 \\
2,117 \\
1,994 \\
2,114 \\
1,992 \\
1,883 \\
1,528\end{array}$ \\
\hline The year............. & 16,900 & 16,300 & 14,000 & 17,800 & 17,600 & 14,600 & 18,300 & 16,200 & 17,500 & 15,000 & 21,000 \\
\hline
\end{tabular}


Sum of monthly discharge over Sunol dam and Brightside weir, in acre-feet.

[Compiled from records of Spring Valley Water $\mathrm{C}_{0 .}$.]

\begin{tabular}{|c|c|c|c|c|c|c|c|c|c|c|c|}
\hline & $1903-4$ & $1904-5$ & $1905-6$ & $1906-7$ & $1907-8$ & $1908-9$ & $1909-10$ & 1910-11 & $1911-12$ & $1912-13$ & $1913-14$ \\
\hline 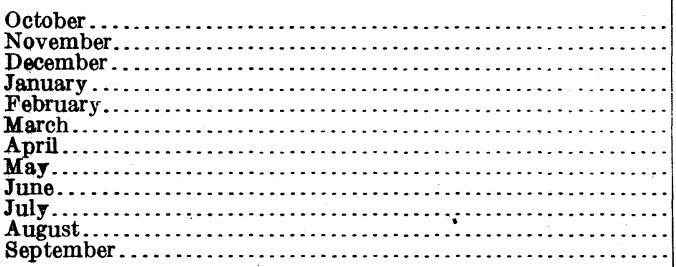 & $\begin{array}{r}1,021 \\
2,588 \\
1,569 \\
1,907 \\
28,885 \\
51,448 \\
17,690 \\
7,113 \\
2,076 \\
1,665 \\
1,456 \\
1,032\end{array}$ & $\begin{array}{r}1,119 \\
1,036 \\
1,908 \\
4,719 \\
12,940 \\
23,008 \\
7,573 \\
6,449 \\
2,013 \\
1,570 \\
1,388 \\
982\end{array}$ & $\begin{array}{r}869 \\
825 \\
882 \\
56,308 \\
23,398 \\
87,934 \\
28,565 \\
8,638 \\
4,668 \\
1,960 \\
1,616 \\
1,547\end{array}$ & $\begin{array}{r}1,414 \\
1,303 \\
21,039 \\
71,146 \\
30,225 \\
172,151 \\
24,262 \\
8,208 \\
4,048 \\
3,427 \\
2,389 \\
1,685\end{array}$ & $\begin{array}{r}1,695 \\
1,975 \\
6,024 \\
15,502 \\
15,343 \\
12,293 \\
3,172 \\
2,410 \\
1,653 \\
1,639 \\
1,221 \\
1,103\end{array}$ & $\begin{array}{r}953 \\
930 \\
1,045 \\
112,022 \\
103,443 \\
22,915 \\
6,265 \\
2,436 \\
1,834 \\
1,778 \\
1,554 \\
1,488\end{array}$ & $\begin{array}{r}1,632 \\
1,471 \\
10,940 \\
35,832 \\
15,955 \\
18,607 \\
8,326 \\
3,391 \\
1,818 \\
1,595 \\
1,599 \\
1,274\end{array}$ & $\begin{array}{r}701 \\
\mathbf{9 6 9} \\
\mathbf{1}, 372 \\
\mathbf{9 5 , 7 9 2} \\
\mathbf{4 3 , 0 7 4} \\
\mathbf{1 2 7 , 3 3 4} \\
\mathbf{7 , 6 4 2} \\
\mathbf{3 , 4 9 3} \\
\mathbf{1 , 9 2 3} \\
\mathbf{1 , 7 9 6} \\
\mathbf{1}, \mathbf{8 9 8} \\
\mathbf{1 , 5 3 5}\end{array}$ & $\begin{array}{r}\mathbf{1}, 696 \\
\mathbf{1}, 675 \\
1,827 \\
\mathbf{5 , 7 3 7} \\
\mathbf{3}, 056 \\
\mathbf{9 , 8 2 0} \\
\mathbf{3 , 2 4 6} \\
\mathbf{1}, \mathbf{9 1 3} \\
\mathbf{1 , 6 1 5} \\
\mathbf{1 , 3 2 2} \\
\mathbf{1 , 1 2 0} \\
\mathbf{9 6 8}\end{array}$ & $\begin{array}{r}948 \\
769 \\
993 \\
6,715 \\
1,566 \\
2,555 \\
1,973 \\
1,697 \\
1,351 \\
1,120 \\
806 \\
1,001\end{array}$ & $\begin{array}{r}941 \\
971 \\
12,040 \\
116,024 \\
53,371 \\
10,215 \\
4,377 \\
2,193 \\
2,179 \\
2,004 \\
1,883 \\
1,528\end{array}$ \\
\hline The year ........... & 118,500 & 64,700 & 217,200 & 341,300 & 64,000 & 256,700 & 102,400 & 287,500 & 3,400 & 21,500 & 207,700 \\
\hline
\end{tabular}


144 CONTRIBUtions to Hydrology of UNITED states, 1914.

\section{GEOLOGIC FORMATIONS IN RELATION TO GROUND WATER.}

\section{OUTIINE OF FORMATIONS.}

The Contra Costa and Mount Hamilton ranges are composed of strata of Jurassic or Lower Cretaceous and later age. They are of complex structure and have been greatly faulted. The major faults, as elsewhere in the Coast Ranges of California, have a northwesterly trend. The older rocks in the mountains are of importance in connection. with the present investigation only in so far as their elevation and topography influence precipitation and run-off, but the Quaternary and some of the Tertiary deposits in the valleys are valuable water bearers.

Geologic formations in the Alameda Creek drainage basin and adjacent areas.

\begin{tabular}{l|l|l}
\hline \multicolumn{1}{c|}{ System. } & \multicolumn{1}{c|}{\begin{tabular}{l}
\multicolumn{1}{c}{ Series. } \\
Formation.
\end{tabular}} \\
\hline Quaternary. & Recent and Pleistocene. & Alluvium. \\
\hline Tertiary or Quaternary. & Santa Clara formation. \\
\hline Tertiary. & Pliocene. & Orinda formation. \\
\cline { 2 - 3 } & Miocene. & Monterey group. \\
\hline Eretaceous. & Eocene. & Tejon formation. \\
\hline Upper Cretaceous. & Chico formation. \\
\hline Lurassic (?). & (?) & Knoxville formation. \\
\hline
\end{tabular}

\section{JURASSIC (?) SYSTEM.}

FRANCISCAN GROUP.

The Franciscan group is characterized by sandstones, bedded radiolarian cherts, and jaspers, greenstones, glaucophane schist, and some other varieties of crystalline schist. It is much shattered and broken, is traversed by many faults, and is cut by many intrusive rocks, chiefly of a basic character and now in part altered to serpentine. The group consists chiefly of sandstone, but the occurrence of the other rocks mentioned gives to the group as a whole a distinctive lithology.

The Franciscan group occupies some 400 square miles of the Alameda Creek drainage basin and covers practically the whole of the basins of Arroyo. Hondo, the upper Alameda, Arroyo del Valle, and Arroyo Mocho. In this large area there are a few small remnants of Cretaceous rocks, suggesting that Cretaceous formations may have covered the whole region at one time but have since been removed by erosion. Smaller outcrops of Franciscan rocks are found elsewhere in the Alameda Creek drainage basin. The Coyote and Little Coyote hills are composed wholly of rocks belonging to that group. 
The age of the group has not been definitely determined but is certainly pre-Knoxville and by most geologists has been assigned to the Jurassic.

CRETACEOUS SYSTEM.

KNOXVILLE AND CHICO FORMATIONS.

The Cretaceous system comprises locally two formations-the Knoxville, or Lower Cretaceous, and the Chico, or Upper Cretaceous. The Knoxville consists largely of shale, whereas the Chico is made up mostly of sandstone and shale with a conglomerate at its base. ${ }^{1}$ The Cretaceous formations occupy a continuous strip extending from a point in the hills east of Berkeley to a point just west and a little south of the south end of Calaveras Valley. This strip is about 40 miles long and ranges in width from less than a mile to more than 6 miles at its widest point just north of Alameda Creek. By far the larger part is occupied by the Chico formation. Besides this large area of Cretaceous rocks there are a number of smaller outcrops.

\section{TERTIARY SYSTEM.}

EOCENE SERIES.

TEJON FORMATION.

The Eocene series is represented by a sandstone that belongs to the Tejon formation and outcrops over a small area in a syncline just west of the crest of Sunol Ridge.

\section{MIOCENE SERIES.}

\section{MONTEREY GROUP.}

The Miocene series is represented by the Monterey group, which occupies a narrow strip that in most places borders the Cretaceous or the Franciscan rocks on the west, but varies in width and position with the structure. In this region the characteristic representative of the group is a siliceous shale much broken and shattered by earth movements and used extensively for road metal.

A prominent formation in the Monterey group is the Briones sandstone, which occupies a considerable area about the crest of the Monument Peak ridge and extends from the vicinity of Alameda Creek on the north to San Felipe Valley on the south. It is characteristically a greenish mottled sandstone carrying abundant marine fossils.

\section{PLIOCENE SERIES.}

ORINDA FORMATION.

The Orinda formation ${ }^{1}$ comprises the older part of the gravel, sand, and clay deposits in the mountain valleys of this region. It is of fresh-water origin and reaches a maximum thickness of more 
than 4,000 feet. $^{1}$ It occupies an area of about 50 square miles just south of Livermore Valley, and a much larger area north of the valley. As the Orinda formation is only partly indurated and comprises much porous material, it is of great importance as a water bearer. Both Branner and Lawson ${ }^{2}$ regard the gravels of this formation as having an immense storage capacity. The catchment area of the Orinda formation tributary to the Livermore Valley, and hence ultimately to the Niles cone, covers more than 100 square miles and ranges from 500 or 600 feet to more than 1,200 feet above sea level. The formation no doubt acts as a high-level reservoir, feeding its water slowly into the lower parts of the valley and thus helping to maintain a higher water level in the more recent gravels that overlie the Orinda in the Livermore and Sunol valleys.

\section{TERTIARY OR QUATERNARY SYSTEM.}

\section{SANTA CLARA FORMATION.}

The Santa Clara is a rather widespread formation of fresh-water origin extending at intervals along both sides of the Santa Clara Valley for almost its entire length. Within the area covered by this report it outcrops over 5 or 6 square miles in the low hills southeast of Irvington and southwest of Mission San Jose. These hills are composed entirely of slightly indurated and poorly assorted sands, clays, and gravels belonging to this formation. The gravels, which are rather dirty, make up considerably less than a third of the formation. The area of outcrop narrows toward the south and the formation pinches out about half a mile south of the Alameda and Santa Clara county line. ${ }^{3}$ That this formation extends some distance into the valley both north and west of the hills near Irvington is indicated by the logs of wells in this vicinity and by the low yield of these wells. The age of the Santa Clara formation is still in question.

\section{QUATERNARY SYSTEM.}

\section{PLEISTOCENE AND RECENT SERIES.}

\section{ALLUVIUM UNDERLYING THE INTERIOR VALIEYS.}

The upper part of the fill in the Livermore and other interior valleys consists of alluvial deposits of the ordinary type. The water level in these deposits is a vital factor in the permanence of the flow of Alameda Creek and hence is an important element in the water supply of the Niles cone. The ground water in the Livermore and Sunol valleys, when not artificially lowered, rises as it approaches

\footnotetext{
1 Branner, J. C., The future water supply of San Francisco from the conservation and use of its present resources, p. 216, Oct. 31, 1912.

2 Branner, J. C., idem pp. 207, 216; Lawson, A. C., Idem, p. 230.

The writer is indebted to Mr. Harold Hannibal for the outlines of this formation.
} 
the rock wall at the entrance of Niles Canyon and thus assists in giving permanence to the flow of the stream. The Livermore Valley is about 350 feet above sea level at its lower end and about 650 feet at its upper end. This gives a slope of about 300 feet in a distance of about 12 miles, or 25 feet to the mile. The water table has a slope that is somewhat more gentle but still sufficient to give the ground water an active movement toward the lower end and to cause it to rise to the surface in that vicinity wherever it finds an opening or wherever the earth is sufficiently porous. This rising water accounts for the swampy lands near Pleasanton.

\section{ALLUVIUM UNDERLYING SANTA CLARA VALIET.}

Santa Clara Valley is covered to a considerable though unknown depth by alluvium. The Niles cone proper and the additional area covered by this report are occupied by this material. The thickness of the alluvium has not been determined, as none of the borings have reached bedrock except in the vicinity of the Coyote Hills, where four or five wells penetrate to solid rock. According to the driller's record, a well on the south bank of Alameda Creek about half a mile east of the upper bridge at Alvarado reaches bedrock at a depth of 266 feet, but this record is probably erroneous, as none of the many other wells in this vicinity are reported to reach bedrock. One well at a distance of about half a mile is 416 feet deep, and another less than a mile away is 528 feet deep and struck good water-bearing gravel at the bottom. All these wells are owned by the Alameda Sugar Co.

Alluvium lies beneath the salt marsh and under the bay itself, as is shown by records of drill holes both on the marsh and in the bay. The fact that gravels are found below the bay is conclusive evidence that the region has sunk with reference to sea level. Some of these gravels are found at depths of more than 400 feet below the surface of the bay. That there has been recent sinking is further shown by the fact that present stream channels may be traced into the bay. The channel of Coyote Creek is distinctly traceable beneath the bay as far north as San Mateo, a distance of about 20 miles from its present mouth. Some of the streams now flowing into the sides of the bay also have well-defined channels extending beneath the bay to the submerged channel of Coyote Creek. (See fig. 9.)

The alluvial deposits were made by streams with very irregular flow, at times subjected to enormous floods and at times carrying little or no water. The sediments were all brought down from the highlands on the east and represent the materials that once occupied the positions of the present mountain canyons.

The mountainous topography of the Alameda Creek drainage basin, its paucity of timber and brush, the seasonal distribution of its 
rainfall, and the concentration of its run-off into a narrow outlet all contribute to make the creek most erratic, and the large extent of this basin gives volume to the floods:

Because of the great floods to which the basin has been subjected and the large amount of water relative to the load of rock waste to be transported, the coarse materials have at times been carried far beyond the mouth of the canyon. This accounts for the abundance of gravels in well sections, even near the bay. These gravels are not in continuous sheets (see Pls. XIV to XVII), nor would this be expected from their mode of origin. They occur in lenses and stringers, which are so abundant that the prospective well owner is almost sure to strike one or more of these beds at no very great depth. Many of the wells are only 50 or 60 feet deep, and wells more than 100 feet deep are rather exceptional.

Streams flowing over alluvial fans are continually changing their courses. They may be slowly eating away their banks on one side while building up on the other, and at the same time they may be depositing gravel in their channels. During later floods these gravels will be buried under finer sediments, thus forming broad lenses or sheets of gravel. At other times these streams break over their banks and may form altogether new channels, the old ones being buried beneath later sediments, thus giving rise to stringer-like deposits.

The Santa Clara Valley is a region of aggradation. At the same time that the stream channels are formed their bottoms are brought to successively higher and higher levels by the deposition of gravel. In times of flood, when the water spreads over a large part of the Niles cone, natural levees are built, and the streams acquire an unstable position, resulting in frequent shifting. Thus hundreds of channels have been born and buried during the upbuilding of the cone.

The old channels diverge from the mouth of the canyons, and, as this is a region of aggradation, one system is superimposed upon another until there is a complex ramification of old channels diverging both horizontally and vertically somewhat like the roots of a tree. These old channels are buried and sealed in deposits of more or less impervious clay, and hence the water that is fed into them from the mass of gravel near the apex of the cone is under pressure in the lower and outer parts of the cone. This pressure gives rise to artesian conditions on the lower parts of the cone and in some places is sufficient to bring the water to the surface and produce flowing wells.

Conditions similar to those of the Niles cone are found over all parts of the Santa Clara Valley, the valley fill having been deposited in a series of coalescent cones or fans. The percentages of the differ- 


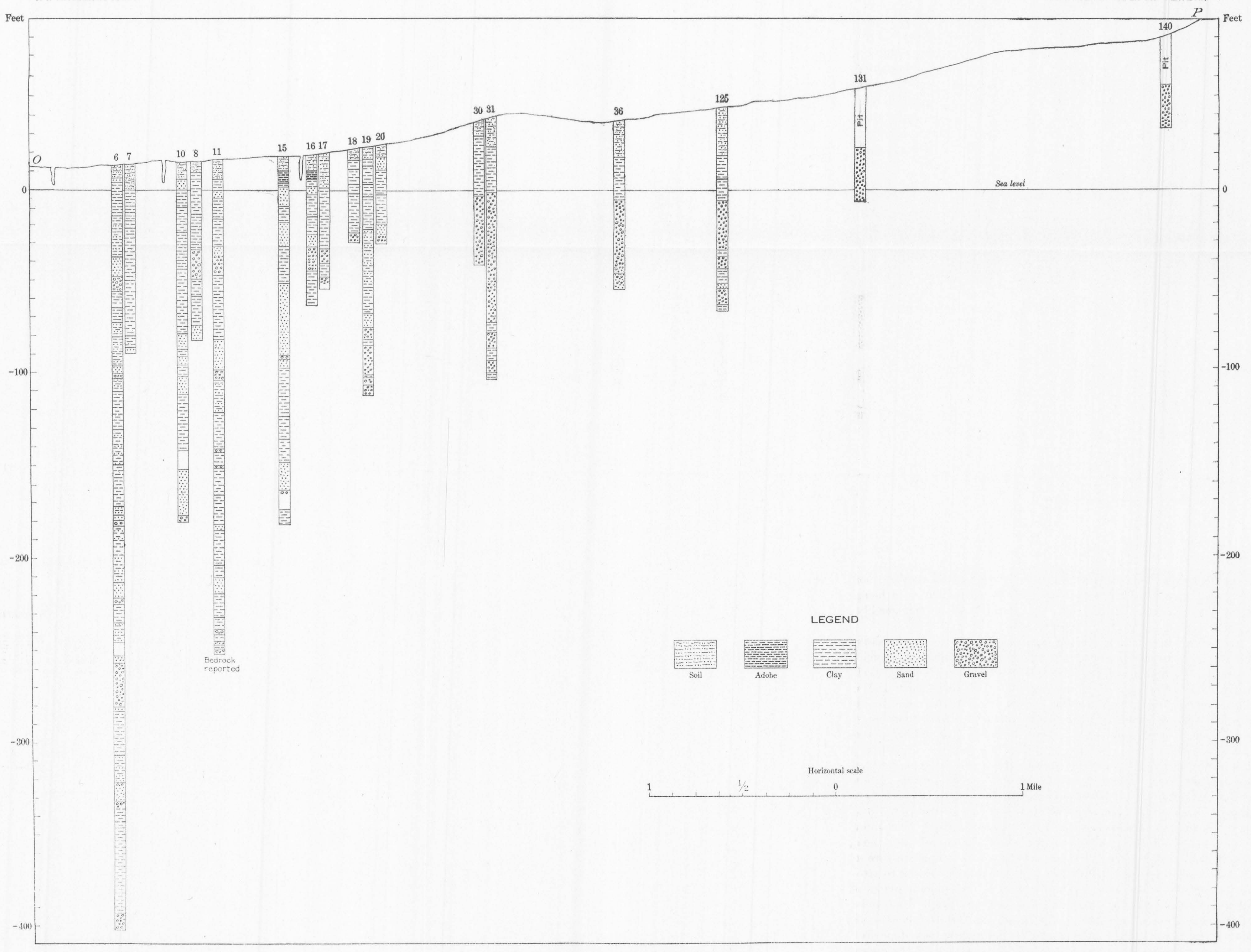


U. S. GEOLOGiCAL SURVEY

WATER-SUPPLY PAPER 345 PLATE XV

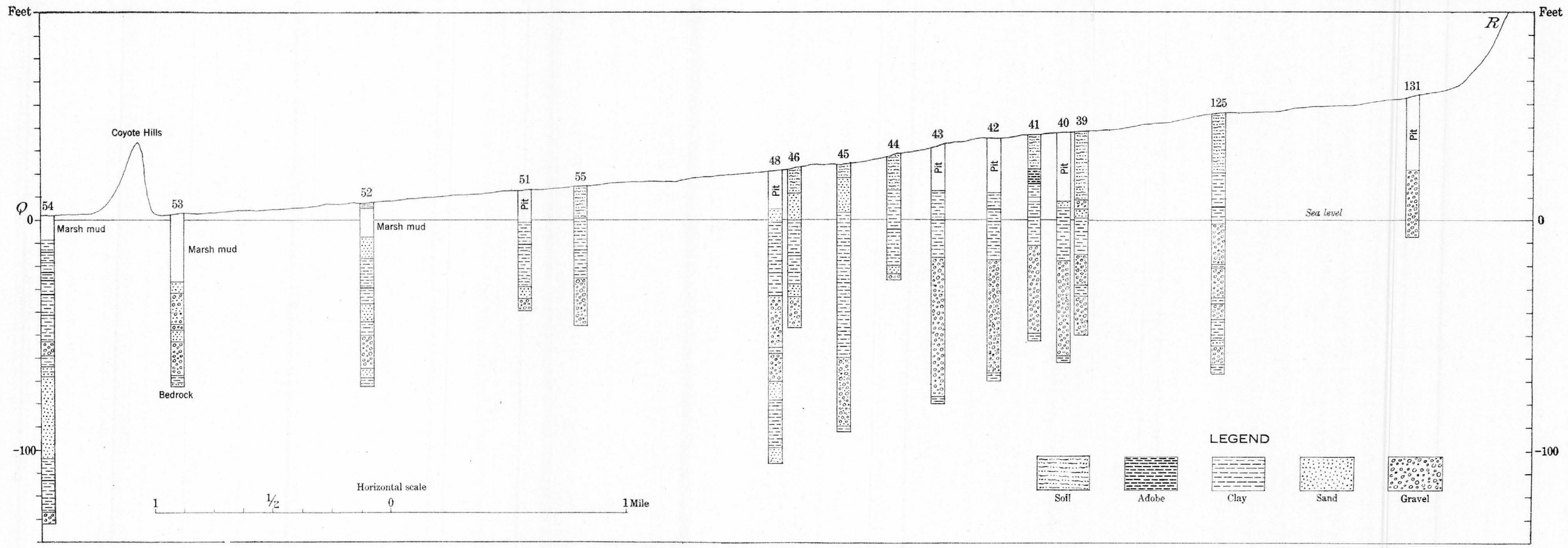

WELL SECTIONS ALONG LINE Q-R PLATE $X$. 


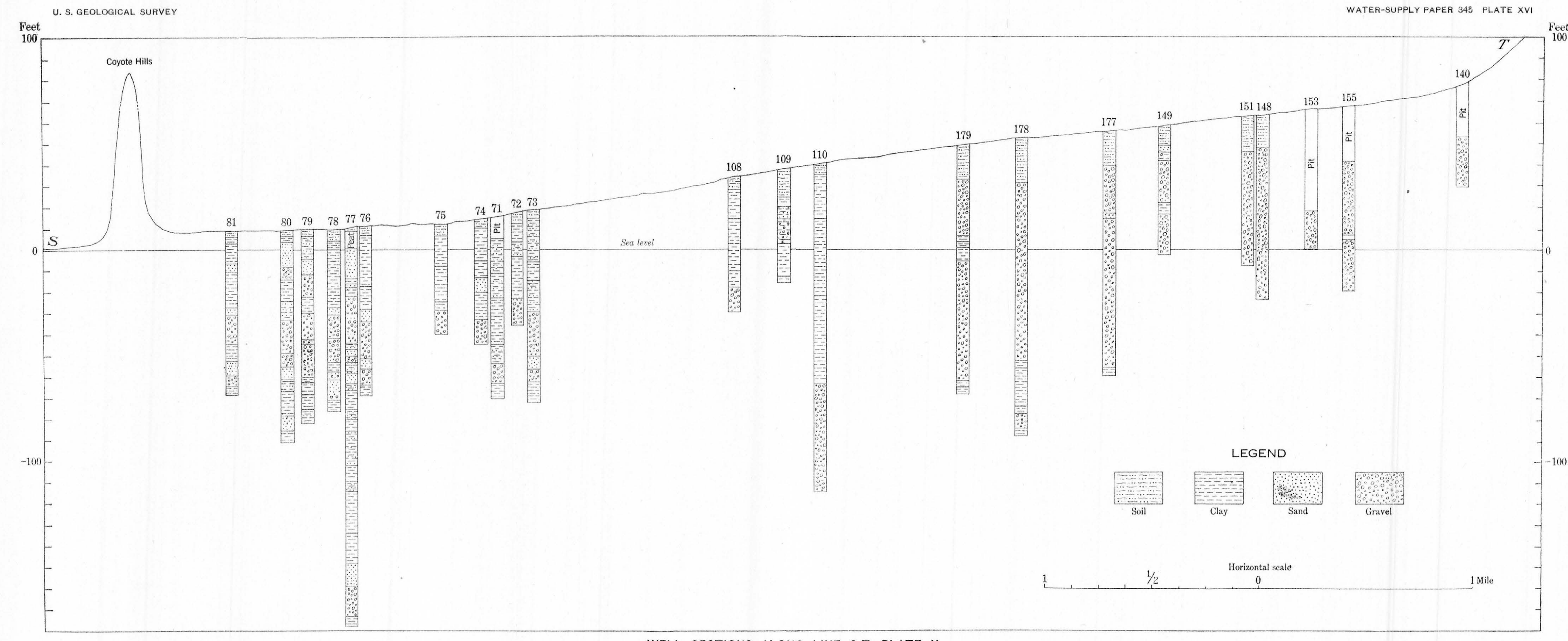




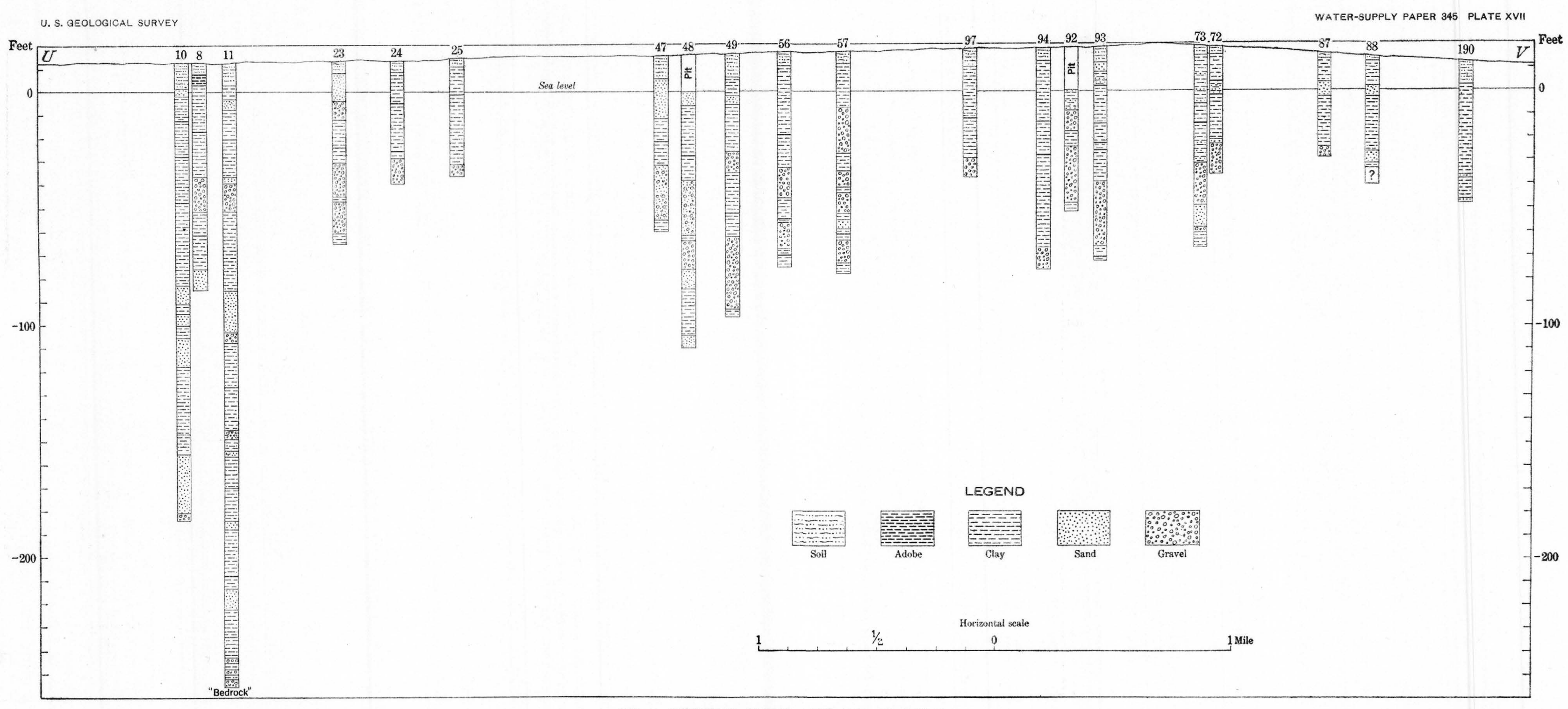

Well sections along line U-V, PLATE $X$. 
ent materials of which the Niles cone and vicinity are composed have been computed from a study of 300 to 400 well logs, with the following result, which is believed to be reasonably accurate:

Estimated composition of the valley fill underlying the Niles cone and adjacent tracts.

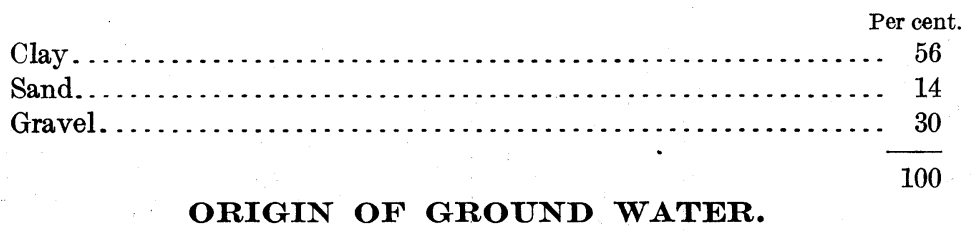

GENERAL SOURCE.

The ground water in the vicinity of Niles has the same source as the surface waters-that is, it originates in the rain that falls upon the drainage basins tributary to this part of the valley and, to a small extent, in the rain that falls upon the valley itself. The question is frequently asked by residents of the district: "Does not our water come from the Sierra?" This question should be answered in the negative. None of the water of the Niles cone and adjacent valley tracts comes from the Sierra Nevada or from any other source outside of the drainage basins tributary to the area.

\section{INFLUENCE OF THE NILES-IRVINGTON FAULT.}

The specific parts of the valley area that are supplied, respectively, by Alameda Creek and by the smaller streams are determined largely by the position of the Niles-Irvington fault. In general the structural and physiographic features of the Coast Ranges of California are controlled by a great system of parallel faults having a northwestsoutheast trend. The Niles-Irvington fault is one of these faults or a branch of one. Between Niles and Irvington it crosses a reentrant of the valley, cutting off the apex of the Niles cone and the flat land between Niles and Mission San Jose. It is plainly marked in its course across the valley land by an eastward-facing escarpment somewhat more than a mile from the base of the mountains. (See Pl. X and Pl. XI, B.) This escarpment is in the valley fill of loose materials and is therefore geologically very young-in fact, it is still growing. Mr. J. C. Shinn reports that during the severe earthquake of 1868 his father's house, standing directly on the fault line, was torn in two and the eastern part dropped about a foot below the western part.

This fault has a profound local effect on the circulation of the ground water as well as on the surface drainage referred to on page 132 of this report. As a result of the displacement the buried gravel channels have apparently been blocked by the impervious materials 
of the valley fill. Consequently the fault plane acts as an underground dam and prevents the passage of the ground water, except in small amounts, from the east side of that plane to the west side. The area between the fault scarp and the base of the mountains receives ground water from all the streams that discharge upon this

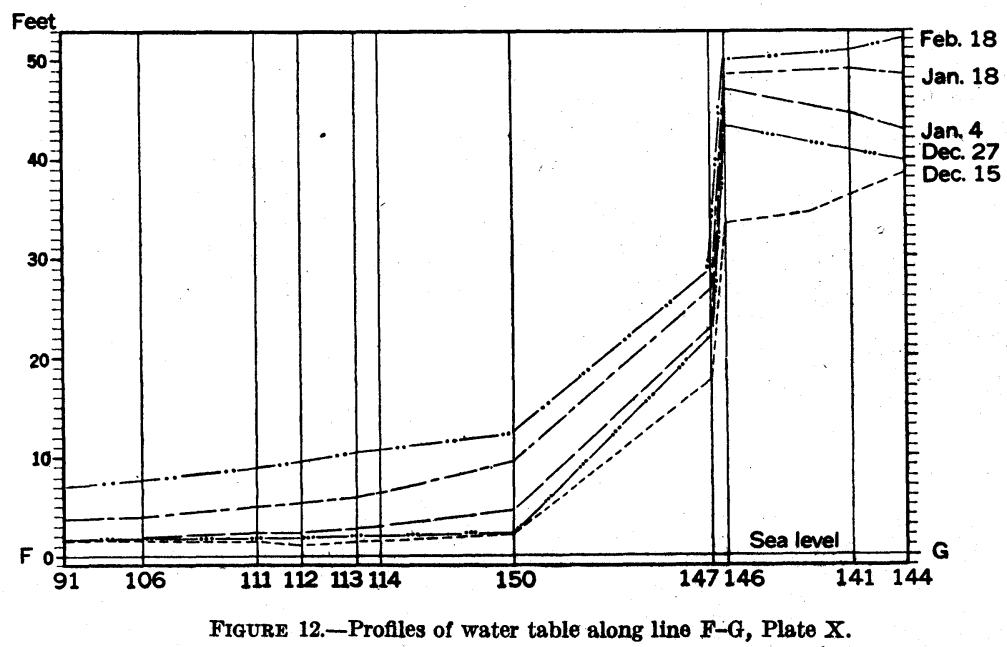

area, but the area west of the fault receives very little ground water except from Alameda Creek, which is the only stream that drains across the fault in the area under discussion. Where the fault cuts through the large gravel deposits near the apex of the Niles cone it is probably less effective as a dam than where the pro-

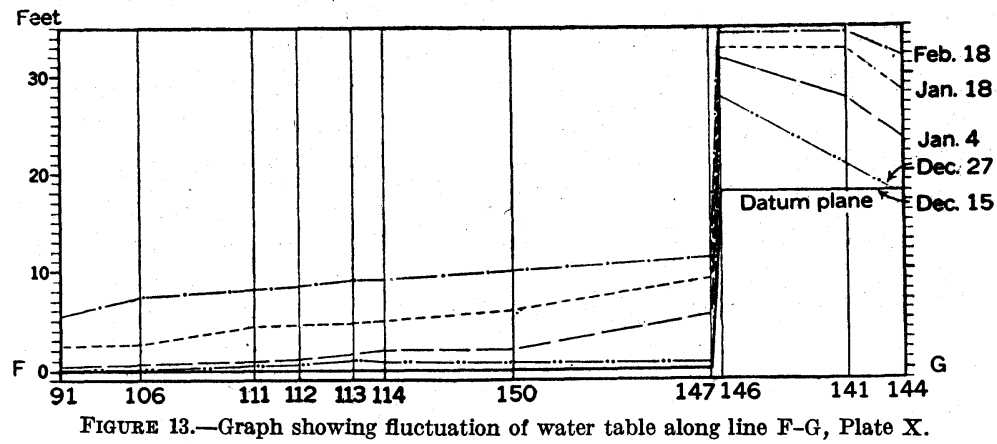

portion of fine sediments is greater, and some of the ground water derived from Alameda Creek may therefore cross the fault through underground channels. Even on the Niles cone, however, the ground water is greatly impounded by the fault, as shown by a difference of more than 20 feet in elevation of the water table at the J. C. Shinn wells. (See table, p. 153.) 


\section{WATER-IEVEL DATA.}

That the Niles-Irvington fault acts as an underground dam substantially preventing the ground water east of the fault plane from crossing to the region west of this plane is proved by the abrupt change in the water table, as shown by the data given in the following tables. This abrupt change is brought out very clearly in the second table (p. 153), in which is given the depth to water in wells arranged in pairs on opposite sides of the fault, and also in Plate $\mathrm{X}$ and in figures 12 to 24 , inclusive. Figures 12 and 20 show profiles based on instrumentally determined levels; the other figures represent fluctuations of the water table by means of graphs. In these graphs the position of the water table on the first date indicated is represented by horizontal lines except at the fault, where the actual differences in level are shown. Later positions of the water table are plotted relative to the horizontal lines. Where sections $\mathrm{DE}, \mathrm{FG}$, and $\mathrm{HE}$ and concentric section $\mathrm{C}_{1}$ cross the fault line the relative elevations of wells on opposite sides were instrumentally determined; ${ }^{1}$ where the other sections, IJ and $\mathrm{KL}$, cross the fault line the differences in elevation were determined by use of a hand level. The relation of the graphs to the profiles is shown by figures 12 and 13 , in which the same data for the section FG are plotted by the two methods.

\section{Depths, in feet, to the water table in the vicinity of Niles.}

[Numbers in parentheses correspond to thase used on Plate $\mathrm{X}$.]

\begin{tabular}{|c|c|c|}
\hline Joseph Furtado (133). & Feb. 17, 1914.. & J. C. Shinn (147). \\
\hline Dec. $10,1913 \ldots \ldots \ldots .15 .75$ & Feb. 24, 1914.. & Nov. $20,1913 \ldots \ldots .51 .50$ \\
\hline Dec. $29,1913 \ldots \ldots \ldots .15 .62$ & J. C. Shinn (138). & Nov. $26,1913 . \ldots \ldots 50.46$ \\
\hline Jan. $18,1914 \ldots \ldots \ldots .11 .12$ & & Dec. $13,1913 .$. \\
\hline Feb. $\quad 4,1914 \ldots \ldots \ldots . .10 .37$ & Nov. 17,1913 (dry). 60.00 & Dec. $23,1913 .$. \\
\hline Feb. $24,1914 \ldots \ldots . . \quad 8.00$ & J. C. Shinn (146). & Dec. $29,1914 \ldots \ldots$... 46.46 \\
\hline County well (132). & Nov. $20,1913 \ldots \ldots . .32 .43$ & Jan. $8,1914 \ldots \ldots \ldots .42 .04$ \\
\hline Nov. 17,1913 (dry). 50.00 & Nov. $26,1913 \ldots$ & Jan. 15, $1914 \ldots \ldots .41 .70$ \\
\hline May 2,1914 (dry). 50.00 & Dec. 13, 1913... & Jan. $22,1914 \ldots \ldots \ldots 40.42$ \\
\hline J. C. Shinn (139). & $\begin{array}{l}\text { Dec. } 27,1913 \ldots \ldots \ldots .22 .70 \\
\text { Dec. } 29,1913 \ldots \ldots \ldots .22 .00\end{array}$ & $\begin{array}{l}\text { Jan. } 27,1914 \ldots \ldots \ldots 39.33 \\
\text { Feb. } 5,1914 \ldots \ldots .38 .58\end{array}$ \\
\hline Dec. $2,1913 \ldots . . . .330 .00$ & Jan. $\quad 4,1914 \ldots$ & Feb. $17,1914 \ldots \ldots .39 .62$ \\
\hline Dec. $12,1913 \ldots \ldots \ldots .29 .83$ & Jan. $\quad 8,1914 \ldots \ldots \ldots .19 .25$ & Feb. $24,1914 \ldots \ldots .38 .62$ \\
\hline Dec. $23,1913 \ldots \ldots \ldots 25.25$ & Jan. $15,1914 \ldots \ldots \ldots$ 17. 96 & Mar. $12,1914 .$. \\
\hline $\begin{array}{lr}\text { Dec. } 28,1913 \ldots \ldots \ldots . & 19.42 \\
\text { Jan. } \quad 2,1914 \ldots \ldots \ldots . & 17.67\end{array}$ & $\begin{array}{l}\text { Jan. } 22,1914 \ldots . \\
\text { Jan. } 27,1914 \ldots .\end{array}$ & H. J. Tilden (155). \\
\hline Jan. $8,1914 \ldots \ldots . .17 .10$ & Feb. $5,1914 \ldots$ & Nov. $7,1913 .$. \\
\hline Jan. $19,1914 \ldots \ldots \ldots .16 .00$ & Feb. 17, 1914... & \\
\hline Jan. $27,1914 \ldots \ldots .12 .21$ & Feb. $24,1914 \ldots$ & H. J. Tilden (154). \\
\hline Feb. $5,1914 \ldots \ldots \ldots .16 .54$ & Mar. $12,1914 \ldots$ & Nov. 17,1913 (dry). 46.60 \\
\hline
\end{tabular}

1 The levels were run for the Spring Valley Water Co. by J. H. Forbes and were kindly made available by the company. 
152 CONTRIBUTIONS TO HYDROLOGY OF UNITED STATES, 1914.

Depths, in feet, to the water table in the vicinity of Niles-Continued.

\section{County well (153).}

Nov. 20, 1913...... 54.92

Nov. 26, $1913 \ldots . . .54 .92$

Dec. 13, 1913...... 55. 02

Dec. $23,1913 . . . . . .55 .08$

Dec. $29,1913 . . . . . .54 .75$

Jan. 3, 1914........ 52.95

Jan. 8, 1914........ 51.25

Jan. 15, 1914........ 50. 04

Jan. 22, 1914........ 48. 58

Jan. 27, 1914........ 47.50

Feb. 6, 1914......... 45. 33

Feb. 18, 1914....... 45.88

M. J. Silva (156).

Dec. 16, 1913....... 28. 52

Dec. 24,1913 .... . . 2 28. 30

Jan. 1, 1914......... 24.80

Jan. 9, 1914........ . 21.90

Jan. 19, 1914........ 18. 54

Jan. 27, 1914........ 15.00

Feb. 11, 1914....... 13. 17

Feb. 18, 1914....... 13. 66

Mar. 2, 1914....... 11.25

Mar. 11, 1914...... 11. 25

\section{G. Duarte (152).}

Nov. $1,1913 . . . . . .57 .50$

Nov. $13,1913 . . . . . .56 .00$

Nov. $30,1913 . . . . .56 .00$

Dec. $12,1913 \ldots . . .56 .25$

Dec. $24,1913 . . . . .554 .00$

Dec. $31,1913 \ldots . . .50 .92$

Jan. 13, 1914 ........ 48.33

Jan. 19, 1914........ 47.00

Jan. $28,1914 . . . . . . .46 .00$

Feb. 1, 1914........ 46. 50

Mar. 3, 1914...... 47. 00

Mar. 11, 1914...... 42.33

\section{S. E. Stivers (157).}

Dec. $1,1913 \ldots . . \ldots 30.02$

Dec. 13, 1913...... 30. 20

Dec. $27,1913 . . . . .30 .30 .04$

Jan. $1,1914 \ldots . . . . .28 .48$

Jan. $9,1914 \ldots . . . .2 .28 .04$

Jan. 19, 1914........ 24. 27

Jan. 27, 1914........ 21. 83

Feb. 11, 1914........ 19. 83

Feb. 18, 1914....... 18. 53

\section{S. E. Stivers (159).}

Dec. $1,1913 . . . . . .20 .38$

Dec. $27,1913 . . . . . .19 .96$
Jan. 1, 1914......... 18. 25

Jan. 9, $1914 \ldots \ldots \ldots$.... 15.62

Jan. 19, 1914........ 12.00

Jan. 27, 1914........ 8. 58

Feb. 11, 1914...... 5.60

Feb. 18, 1914...... 5.36

\section{S. E. Stivers (160).}

Dec. 16, 1913 ...... 22.33

Dec. $24,1913 \ldots . . .22 .06$

Dec. $27,1913 . . . .21 .37$

Jan. 2, 1914....... 18. 80

Jan. 9, 1914 . ....... 16. 46

Jan. 19, 1914........ 13.00

Jan. 27, 1914........ 9. 90

Feb. 11, 1914....... 7.33

Feb. 18, 1914....... 6.72

Mar. 2, 1914....... 4.17

Mar. 8, 1914....... 4. 00

\section{S. E. Stivers (161).}

Dec. $16,1913 . . . . . .59 .17$

Dec. $27,1913 . . . . .559 .64$

Jan. 2, 1914 ....... 58. 90

Jan. 9, 1914........ 58. 17

Jan. 19, 1914. . . . . . . 57. 12

Jan. 27, 1914........ 55.90

Feb. 11, 1914....... 55. 53

Feb. 18, 1914....... 54.43

Mar. 2, 1914....... 51. 40

Mar. 8, 1914....... 50. 50

\section{A. G. Peixoto (162).}

Nov. $7,1913 . . . \ldots .54 .77$

Dec. $1,1913 \ldots \ldots . .56 .00$

Dec. $27,1913 . . . . .55 .20$

Jan. 2, 1914 . ...... 55.00

Jan. $12,1914 \ldots . . . . .54 .50$

Jan. 21, 1914........ 53. 75

Jan. 27, 1914........ 53. 12

Jan. 31, 1914........ 52. 70

Feb. 5, 1914......... 51. 92

Feb. 24, 1914...... 50. 00

Mar. $4,1914 . . . . .50 .00$

\section{J. S. Leal (164).}

Dec. $27,1913 \ldots . .662 .30$

Jan. $2,1914 \ldots . .662 .25$

Jan. 12,1914 . ..... . 61. 96

Jan. 21, 1914........ 61.33

Jan. 27, 1914........ 60. 83

Jan. 31, 1914........ 60. 50

Feb. 5, 1914....... 59. 92

Feb. $24,1914 \ldots . . .58 .00$
J. F. Nunes (172).

Nov. 20, 1913.... . . 50.17

Nov. 26, 1913....... 50. 20

Dec. $13,1913 \ldots . . .50 .54$

Jan. 1, 1914......... 50. 54

Jan. $12,1914 . . . . . . .50 .50$

Jan. 21, 1914........ 49.96

Jan. 31, 1914........ 49. 20

Feb. $6,1914 \ldots . . . . . .48 .33$

Fieb. 24, 1914....... 46.66

California Nursery (165).

Nov. $15,1913 . . . \ldots .6 .24$

Jan. 9, 1914....... Flooded.

- Kirk (166).

Nov. $7,1913 \ldots . . . .34 .29$

Jan. 28, 1914........ 22. 00

Feb. 10, 1914...... 21.00

Mar. 2, 1914....... 19. 00

M. G. Peixoto (168).

Nov. $7,1913 \ldots . . .64 .69$

Dec. $4,1913 \ldots . . . . .67 .00$

Dec. 15,1913 . . . . . 66. 66

Dec. $22,1913 \ldots . .68 .00$

Dec. $27,1913 \ldots . .667 .33$

Dec. $29,1913 \ldots . .668 .00$

Jan. 1, 1914....... 67.50

Jan. 5, 1914........ 68.00

Jan. 12, 1914....... 67. 83

Jan. $26,1914 \ldots . . .665 .50$

Feb. $4,1914 \ldots . . .666 .00$

Feb. 16, 1914.......66.00

J. F. Brown (169).

Dec. 27,1913 ...... 73.42

Jan. $1,1914 . . . \ldots . .72 .46$

Jan. 9,1914 ......... 71.55

Jan. 19, 1914 ........ 69.70

Jan. 27, $1914 \ldots \ldots . .67 .44$

Jan. 31,1914 ....... 66.62

W. Hearsh (167).

Dec. $13,1913 \ldots \ldots .56 .56$

Dec. $27,1913 . . . \ldots 56.10$

Jan. $1,1914 . . . . . . .55 .46$

Jan. 9, $1914 \ldots . . . . .54 .93$

Jan. 19, $1914 \ldots . . .53 .68$

Jan. 27, 1914 ...... 52.00

Jan. $31,1914 . . . . .51 .38$ 
Depth to the water level in wells arranged in pairs on opposite sides of the Niles-Irvington fault.

[The location of wells is shown on Plate $\mathrm{X}$ by corresponding numbers.]

\begin{tabular}{|c|c|c|c|c|c|c|c|}
\hline $\begin{array}{l}\text { No. of } \\
\text { well. }\end{array}$ & Owner. & $\begin{array}{l}\text { Side of } \\
\text { fault. }\end{array}$ & $\begin{array}{l}\text { Depth of water } \\
\text { level below } \\
\text { surface. }\end{array}$ & $\begin{array}{c}\text { Differ- } \\
\text { ence in } \\
\text { elevation } \\
\text { of surface. }\end{array}$ & $\begin{array}{l}\text { Differ- } \\
\text { ence in } \\
\text { water } \\
\text { level. }\end{array}$ & $\begin{array}{l}\text { Approxi- } \\
\text { mate } \\
\text { distance } \\
\text { apart. }\end{array}$ & $\begin{array}{c}\text { Date of } \\
\text { measure- } \\
\text { ment } \\
(1913) .\end{array}$ \\
\hline $\begin{array}{l}132 \\
133\end{array}$ & $\begin{array}{l}\text { Road well....... } \\
\text { Joseph Furtado. }\end{array}$ & $\begin{array}{l}\text { West.. } \\
\text { East.. }\end{array}$ & $\begin{array}{l}\text { Feet. } \\
\text { Dry at } 50 \\
\quad 25.50\end{array}$ & $\begin{array}{l}\text { Feet. } \\
a_{2}\end{array}$ & $\begin{array}{l}\text { Feet. } \\
23.50+\end{array}$ & $\begin{array}{l}\text { Feet. } \\
\quad 100\end{array}$ & Nov. 17 \\
\hline $\begin{array}{l}138 \\
139\end{array}$ & 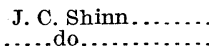 & $\begin{array}{l}\text { West. } \\
\text { East.. }\end{array}$ & $\begin{array}{r}\text { Dry at } 59.89 \\
26.57\end{array}$ & $a 5$ & $28.23+$ & 125 & Do. \\
\hline $\begin{array}{l}147 \\
146\end{array}$ & 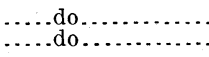 & $\begin{array}{l}\text { West.. } \\
\text { East. }\end{array}$ & $\begin{array}{l}53.81 \\
31.40\end{array}$ & 2.31 & 20.10 & 150 & Nov. 7 \\
\hline $\begin{array}{l}154 \\
155\end{array}$ & $\begin{array}{l}\text { H. J. Tilden... } \\
\text {......do.......... }\end{array}$ & $\begin{array}{l}\text { West.. } \\
\text { East.. }\end{array}$ & $\begin{array}{r}\text { Dry at } 46.60 \\
28.34\end{array}$ & $a_{0}$ & $18.26+$ & b 500 & Do. \\
\hline $\begin{array}{l}161 \\
160\end{array}$ & $\begin{array}{c}\text { S. E. Stivers... } \\
\text {.....do................ }\end{array}$ & $\begin{array}{l}\text { West. } \\
\text { East.. }\end{array}$ & $\begin{array}{l}58.58 \\
22.38\end{array}$ & 4.29 & 31.91 & 200 & Nov. 15 \\
\hline $\begin{array}{l}168 \\
166\end{array}$ & $\begin{array}{r}\text { M. G. Peixoto.. } \\
\text { Kirk..... }\end{array}$ & $\begin{array}{l}\text { West.. } \\
\text { East.. }\end{array}$ & $\begin{array}{l}64.19 \\
33.79\end{array}$ & $a 8$ & 22.40 & 300 & Do. \\
\hline
\end{tabular}

$a$ Approximate.

$b$ These wells are both very near the fault line, the distance between them being chiefly along the fault.

\section{INTERPRETATION OF THE DATA.}

The data given in the preceding tables show that practically all the ground water on the west side of the Niles-Irvington fault, both

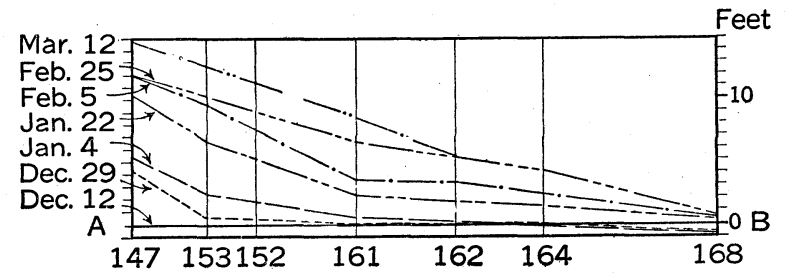

FIGURE 14.-Graph showing fluctuation of water table along line A-B, Plate X.

on the Niles cone and on the adjacent area to the south ( $\mathrm{Pl} . \mathrm{X}$ ), comes from Alameda Creek, the area east of the fault being supplied

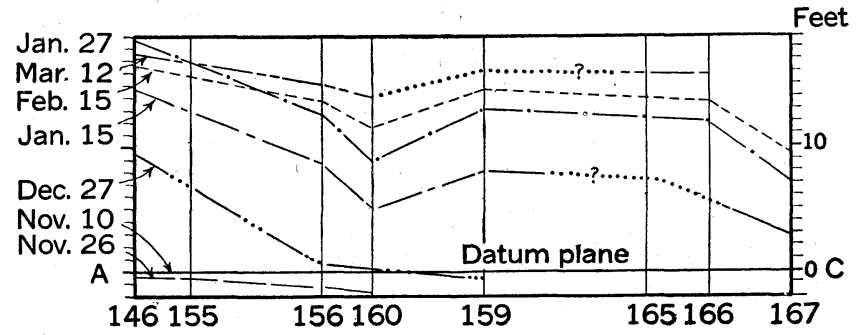

FIGURE 15.-Graph showing fluctuation of water table along line A-C, Plate X.

in the north by Alameda Creek and farther south by the smaller streams that emerge from the mountains south of this creek. If 
wells on opposite sides of the fault receive water from the same source there should be no appreciable difference in the time in which they show response to rainfall, and whatever slight lag the wells on the west side might show should be as much in the northern as in the southern part of the area. If, however, as stated, the wells on the

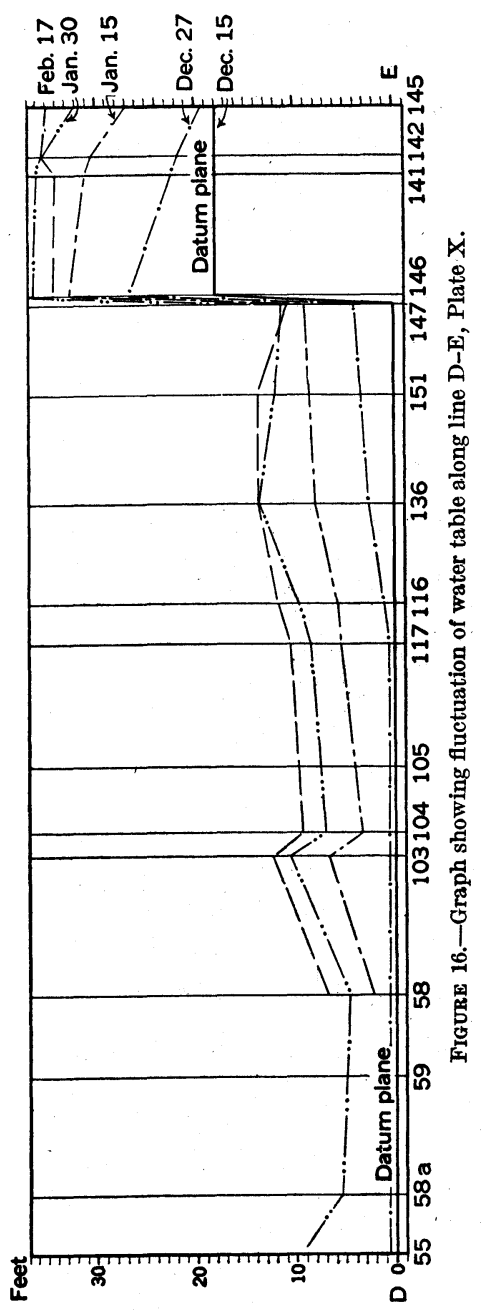
east side are fed not only by Alameda Creek but also by the small streams farther south, while the wells on the west side are supplied only from Alameda Creek, thereshould beconsiderable difference south of the Niles cone in the time in which two wells just across the fault line from each other respond to rainfall. Moreover, this difference in time of response should increase toward the south, because the ground water on the west side must travel successively longer and longer distances on its journey southward. That this lag really occurs and that it is progressive southward is abundantly demonstrated by the data in the first table and by figures 14 and 15 .

Section AB (fig. 14, the method of construction of which was given on p. 151) represents the positions of the water table on the dates indicated relative to its position on December 12, 1913. Up to that time the water table had been going down, though slowly, for a month or two previous. So far as this section is concerned, December 23 might as well have been selected for the date of beginning, for the water in well 147 was then 50.38 feet below the point of measurement, while on December 12 it was 50.60 feet below the same point. On the afternoon of December 23 Alameda Creek began flowing for the first time during the season. On December 26 the water in well 147 had risen about 2 feet, and on December 29, as shown in the graph (fig. 14), it had risen more than 4 feet. This response was, however, shown only a very short distance to the south, the water level in well 153 having risen only about 6 
inches on December 29. On January 4 the water in well 147 showed a further rise, and the response extended considerably farther south, though even at this date it practically died out within a distance of 1 mile. Meanwhile the water table at the south end of the section

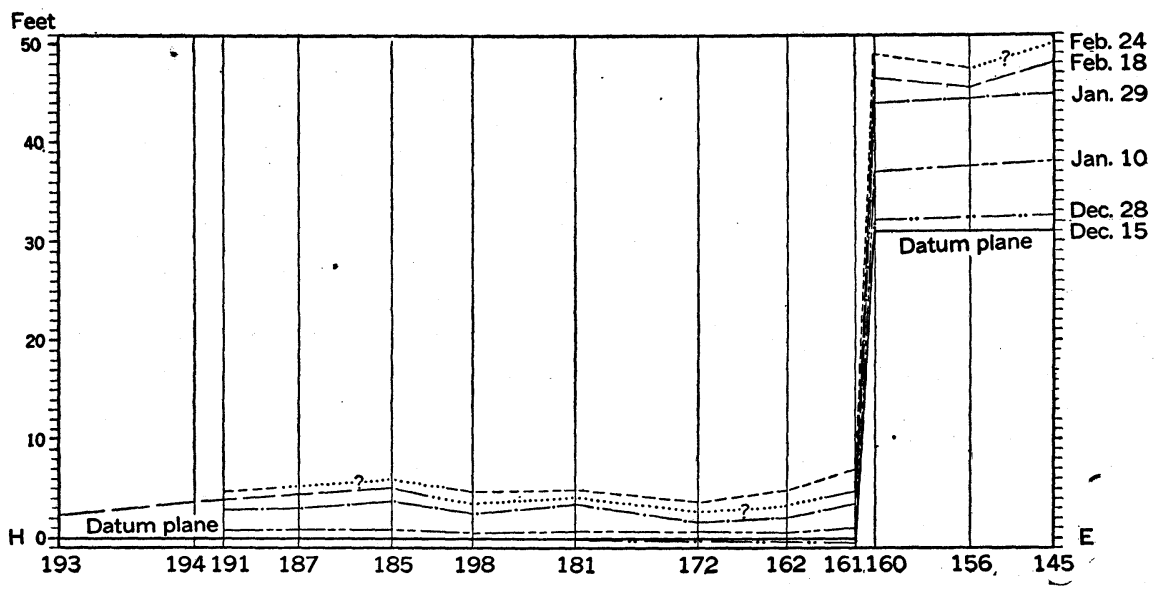

Fraure 17.-Graph showing fluctuation of water table along line H-E, Plate X.

shown in figure 14 continued to sink slowly. The curve for each successive date shows the same slow progress toward the south.' By studying the graph one may almost see the water creeping slowly southward from Alameda Creek. Even as late as March 12 the rise

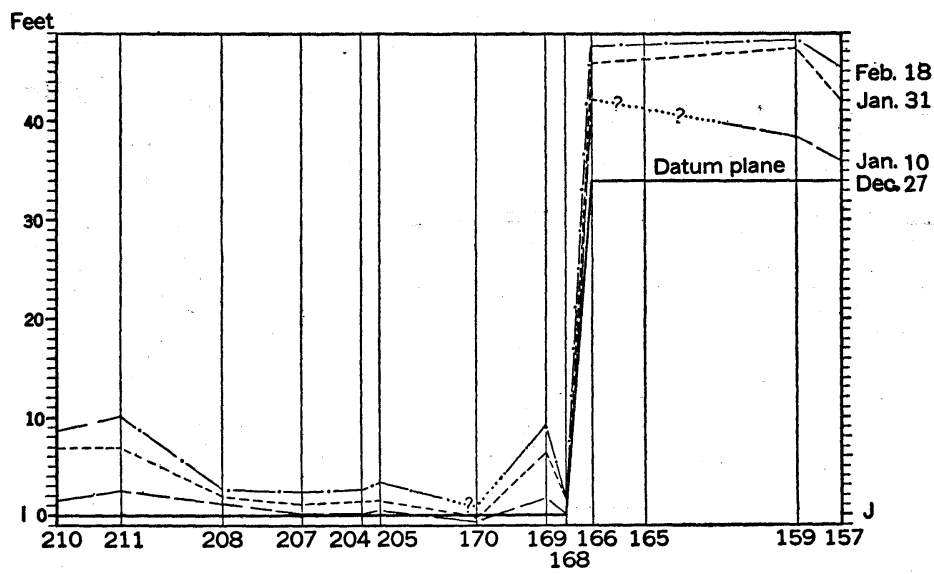

FIGURE 18.-Graph showing fluctuation of water table along line I-J, Plate X.

at the south end was almost negligible, although at the north end it amounted to nearly 15 feet.

Section AC, just east of the fault (fig. 15), is of very different character from section $A B$, just west of the fault (fig. 14). In 
section AC water is supplied from several sources, as is shown by the depressions between different drainage areas, but in section $A B$ the water comes from a single source. These two sections are very close together in most places (Pl. X). At their north ends they

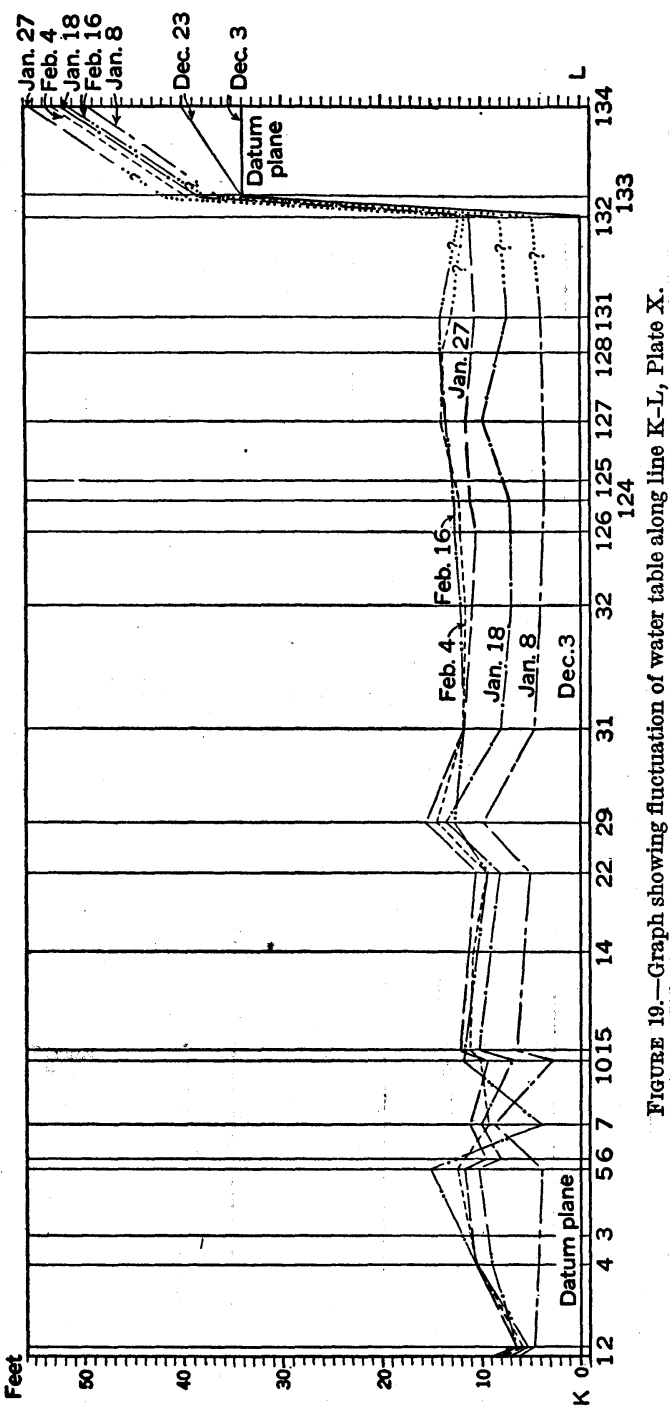
start from practically the same point and at the south end of $\mathrm{AB}$ they are only about 300 feet apart.

A well (169) just south of section $\mathrm{AB}$ showed a quick response to rainfall and a very much greater rise than its nearest neighbor to the north, about 500 feet distant. This difference is thought to be due to a small leak through the fault plane near the well. The leakage, however, is not sufficient to affect neighboring wells and may affect this one simply because of its nearness to the point of lenkage. The water contours for Docember (Pl.X) mightsuggest that there was a considerable leak through the fault dam where it crosses Alameda Creek, but the crowding of the contours at this point is thought to be due in large part to the fact that the Niles Sand \& Gravel \& Rock Co. is continually pumping water and turning it down the creek. This water flows for some distance beyond the fault dam and then rapidly disappears.

The profiles of the water table in section MN (fig. 20), which are based on levels instrumentally determined, show the true slope of the water table along this line and therefore the direction of movement of the ground water, or, rather, not the path of actual motion 
but the line of one of the two components into which the true motion can be resolved. The absolute motion at any given place, other things being equal, is determined by the direction of steepest slope. As surface water runs down a hill in the direction of steepest slope instead of following some line having less slope, just so ground water tends to move along the path of steepest slope unless friction in this direction is so much greater that it neutralizes the effects of steeper slope. The exact position of this path of steepest slope can not be determined from a single section, but the single section $\mathrm{MN}$, for example, shows that one component of the motion of the water is southward.

The four concentric sections, $\mathrm{C}_{1}, \mathrm{C}_{2}, \mathrm{C}_{3}$, and $\mathrm{C}_{4}$, shown in figures 21 to 24 , although not controlled by levels, have the same general character as section MN. The general slope in each is toward the south, and the irregularities of this slope are alike in character, occurring where they would be expected on account of character of the soil or relation to the drainage. These sections also show the lines of greatest flow and therefore of least resistance. They show two maxima-one along the present Alameda Creek, the other along "Old Alameda Creek," or Sanjen de los Alisos-with a strong movement throughout the whole area lying between them. On either side of this interstream area the slope becomes more gentle and more nearly uniform. In the region between wells 205 and 207 , however, the slope is abruptly reversed, indicating flow in a northerly direction. This is seen on four of the five profiles in section MN (fig. 20). The profile for December 23 shows practical stagnation in the region between wells 116 and 205, but with a slight backward drift of the water. This movement is also indicated by the water contours for December shown on Plate X.

The slight backward drift shown in this region is believed to be due to the heavier pumping in the more central portions of the Niles

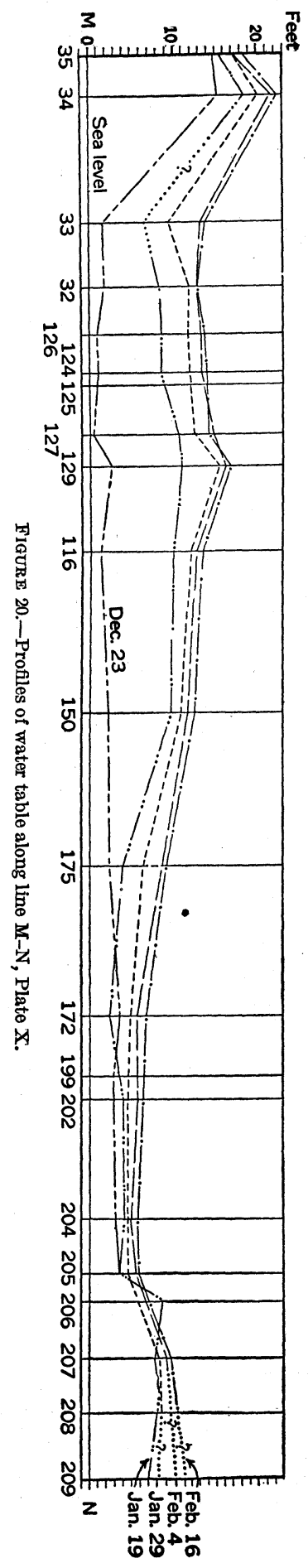


cone, and especially that of the People's Water Co. at Alvarado. As shown by later graphs and profiles and also by the water contours for February (Pl. X), the water in the region between Alameda Creek and well 205 comes from a northerly direction. But during the lowwater season, and especially when there is heavy pumping to the north, the water table in this vicinity will be lowered so much that a slope toward the region of heavy pumping will be established and consequently a reversal of direction of movement of ground water will occur.

The more abrupt slope near Niles was probably caused by the pumpage of the Niles Sand \& Gravel \& Rock Co. (p. 156) and perhaps by slight leakage through the fault dam in the vicinity of Alameda

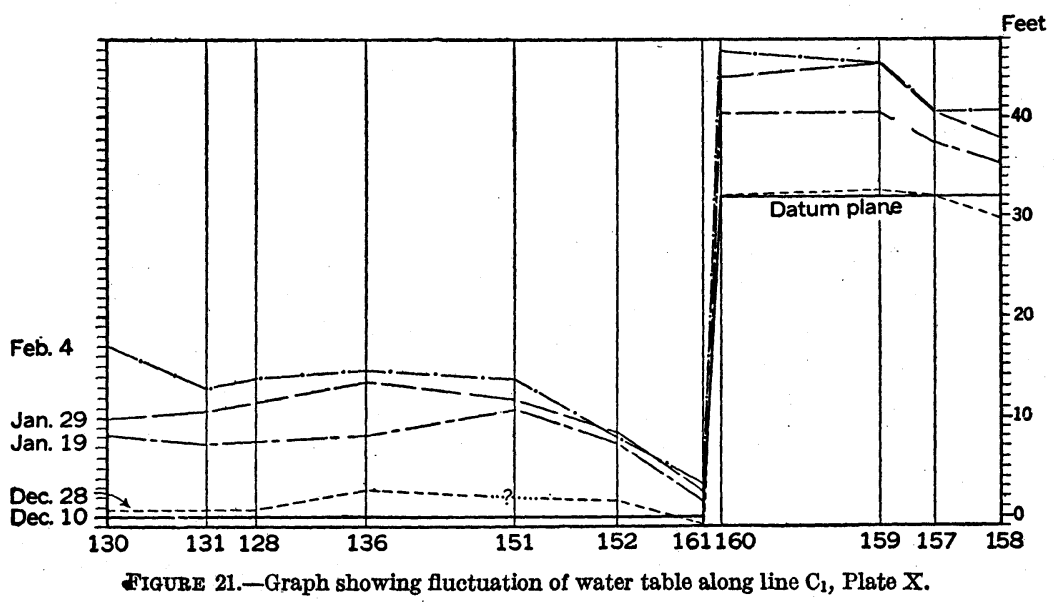

Creek. The slope near Alvarado was caused by heavy pumping by the People's Water Co.

The conclusions reached are that the portions of Niles cone and the adjacent tract to the south covered by this report lying west of the fault receive their water from Alameda Creek, and that the area east of the fault receives its water partly from Alameda Creek and partly from the smaller streams that discharge upon this area.

\section{CONTRIBUTIONS TO THE GROUND-WATER SUPPLY.}

The amount of ground water annually used can not exceed the difference between the amount added to the ground-water supply during an average season and the amount unavoidably lost each year. Indeed the yield on which it is safe to count is somewhat less than this difference, as it depends to a large extent on the more unfavorable seasons. The amount of water percolating downward and joining the ground water in an area such as is here described depends on 
a number of conditions, some of the most influential of which are the size and character of the drainage basins tributary to the area, the amount and distribution of the rainfall in these drainage basins, the topography of the area, the permeability and porosity of the materials underlying the area, and the elevation of the water table at the beginning of the replenishing season. In any given area all these conditions are constant except the rainfall, its distribution, and the position of the water table. A large amount and proper distribution of rainfall and a low position of the water table are favorable to groundwater storage.

An estimate was made of the amount of water added during the season of 1913-14 to the underground supply of the Niles cone and that part of the additional tract outlined on page 127 lying west of the fault. This estimate was made by multiplying the average rise of the water table, in feet, by the percentage of pore space, and this product by the area, in acres, the result being expressed in acre-feet. The average rise of the water table was obtained by averaging the measured rise in 125 wells subequally distributed over the entire area west of the fault and found to be 11.32 feet. The porosity was assumed to be 25 per cent. The area was estimated, by measuring with a planimeter on a photographic enlargement of the United States Geological Survey topographic maps, at 20,817 acres. An estimate based on these figures gives the supply 


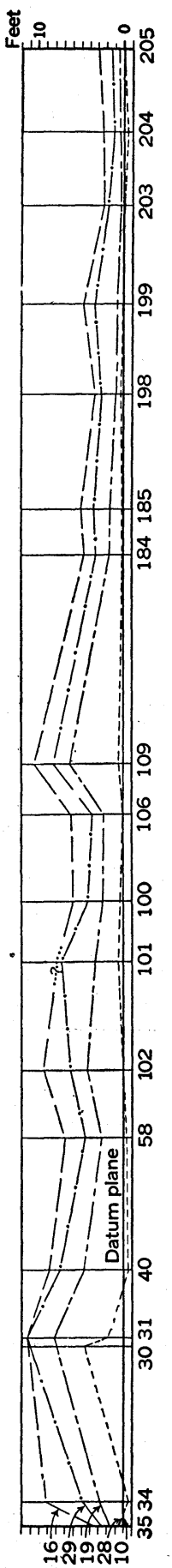

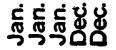

added during the season of 1913-14 as 58,912 acre-feet, exclusive of the amount withdrawn during the period of rising water.

The assumption of 25 per cent porosity is believed to be within reasonable limits of error. Mendenhall ${ }^{1}$ in his studies of the San Joaquin Valley assumed a porosity of 20 per cent, but the materials of the San Joaquin Valley are finer on the average than those dealt with here, and their available pore space is doubtless less. Buckley ${ }^{2}$ determined the maximum porosity of sandstone at more than 28 per cent. T. W. Espy ${ }^{3}$ made porosity determinations of soils in the Livermore Valley ranging from 27.3 to 49.5 per cent, with a general average of 37.6 per cent, but it is believed that his method, that of pouring the soil into water and shaking, yields too high results. Lee ${ }^{4}$ in certain tests with materials in Owens Valley found approximately 28 per cent of pore space. The materials in these tests were dry gravel, sand, and silt, which probably have more pore space than the average sediments of the Niles cone region.

The rapidity with which the materials underlying the Niles cone absorb water is indicated in the following table: ${ }^{5}$

\footnotetext{
1 Mendenhall, W. C., Preliminary report on the ground waters of the San Joaquin Valley, Cal.: U. S. Geol. Survey Water-Supply Paper 222, p. 27, 1908.

2 Buckley, E. R., Building and ornamental stones of Wisconsin: Wisconsin Geol, and Nat. Hist. Survey Bull. 4, pp. 400-403, 1898.

3 The future water supply of San Francisco from the conservation and use of its present resources, p. 202, Oct. 31, 1912.

4 Lee, C. H., An intensive study of the water resources of a part of Owens Valley, Cal.: U. S. Geol. Survey WaterSupply Paper 294, diagram opposite p. 82, 1912.

5 Lippincott, J. B., California hydrography: U. S. Geol. Survey Water-Supply Paper 81, p. 33, 1903. Measurements made by W. W. Brier.
} 
Discharge measurements of Alameda Creek, Alameda County, Cal.

\begin{tabular}{|c|c|c|c|c|c|}
\hline \multirow[b]{2}{*}{ Date. } & \multicolumn{2}{|c|}{ Discharge in second-feet. } & \multirow[b]{2}{*}{ Date. } & \multicolumn{2}{|c|}{ Discharge in second-feet. } \\
\hline & $\begin{array}{l}\text { Niles dam, } \\
\text { about } 1 \frac{1}{2} \\
\text { miles above } \\
\text { Niles. }\end{array}$ & $\begin{array}{l}\text { Three miles } \\
\text { below Niles. }\end{array}$ & & $\begin{array}{c}\text { Niles dam, } \\
\text { about } 1 \frac{1}{2} \\
\text { miles above } \\
\text { Niles. }\end{array}$ & $\begin{array}{l}\text { Three miles } \\
\text { below Niles. }\end{array}$ \\
\hline $\begin{array}{l}\text { Dec. } 18,1896 \ldots \ldots \ldots \\
\text { Dec. } 19,1896 \ldots \ldots \ldots \\
\text { Dec. } 20,1896 \ldots \ldots \ldots\end{array}$ & $\begin{array}{l}38.68 \\
32.49 \\
30.94\end{array}$ & $\begin{array}{r}7.74 \\
6.19 \\
.77\end{array}$ & $\begin{array}{l}\text { Jan. } 11,1897 \ldots \ldots \ldots \ldots \\
\text { Jan. } 12,1897 \ldots \ldots \ldots \ldots \\
\text { Jan. } 18,1897 \ldots \ldots \ldots \ldots\end{array}$ & $\begin{array}{l}63.44 \\
57.25 \\
51.06\end{array}$ & $\begin{array}{l}44.87 \\
40.23 \\
34.04\end{array}$ \\
\hline
\end{tabular}

The rainfall during the season of 1913-14 was somewhat above the normal, as is shown by the following table. Moreover, it was well distributed, as is indicated by the monthly records given on pages 135-141. The large and well-distributed rainfall and the low groundwater levels at the beginning of the season tended to make the additions to the ground-water supply in this year unusually large.

Total precipitation July 1, 1918, to June 30, 1914, and seasonal averages for stations in the vicinity of Niles.

\begin{tabular}{|c|c|c|c|}
\hline \multirow[b]{2}{*}{ Station. } & \multirow[b]{2}{*}{$\begin{array}{c}\text { Elevation } \\
\text { above sea } \\
\text { level. }\end{array}$} & \multicolumn{2}{|c|}{ Precipitation. } \\
\hline & & $\begin{array}{l}\text { July 1, } \\
1913, \text { to } \\
\text { June 30, } \\
\text { 1914, in- } \\
\text { clusive. }\end{array}$ & $\begin{array}{l}\text { Seasonal } \\
\text { average. }\end{array}$ \\
\hline 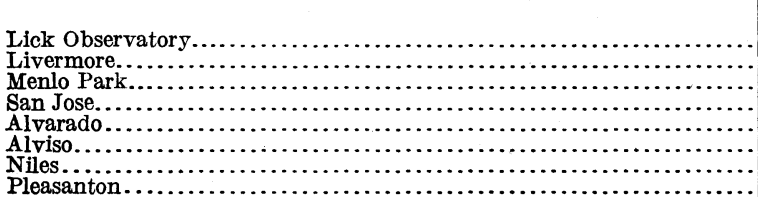 & $\begin{array}{r}\text { Feet. } \\
4,209 \\
485 \\
64 \\
95 \\
19 \\
11 \\
87 \\
355\end{array}$ & $\begin{array}{r}\text { Inches. } \\
35.61 \\
17.01 \\
24.12 \\
19.45 \\
20.35 \\
16.44 \\
18.91 \\
17.05\end{array}$ & $\begin{array}{r}\text { Inches. } \\
31.42 \\
15.49 \\
a 16.85 \\
15.09 \\
17.64 \\
15.01 \\
18.61 \\
16.03\end{array}$ \\
\hline
\end{tabular}

a Seasonal average does not include 1913-14.

The seasons 1911-12 and 1912-13 were probably the two successively driest seasons on record, and the preceding season (1910-11) was also unfavorable because more than half of the rain of the entire season fell during the month of January. This period represents one of the most trying droughts the country has experienced and one in which the dependence on ground water was very great. However, a number of seasons on record had no greater precipitation than that of 1912-13. During the season of 1912-13 the ground water did not rise at all over a large part of the area and rose only from a fraction of a foot to 18 inches or 2 feet over the rest of it. The total amount of water added during this season, exclusive of withdrawals during the replenishing period, is estimated, from the data at hand, at only 2,600 acre-feet. 
It is concluded that the two years covered by the investigation, 1912-13 and 1913-14, represent nearly extreme conditions, and that the range in annual contribution of ground water to the Niles cone and adjacent area west of the fault is between 2,600 and 59,000 acrefeet, or between somewhat wider limits, exclusive of the withdrawals during the winter period of rising ground water. The range in ground-water contributions is therefore relatively much greater than the range in precipitation.

\section{WITHDRAWAL OF GROUND WATER. SPRING VALIEY WATER CO.}

About 16 million gallons a day, ${ }^{1}$ or 18,000 acre-feet a year, is withdrawn by the Spring Valley Water Co. from the Alameda Creek drainage basin above Niles for the public supply of San Francisco. If this water were not diverted a large part of it would ultimately reach the underground reservoir of the Niles cone. (See p. 135.) Twothirds of this amount would be equivalent, on the assumption of 25 per cent porosity, to a rise of the water table of about $2 \frac{1}{4}$ feet a year.

\section{PEOPLE'S WATER CO.}

The People's Water Co. is withdrawing about 8 million gallons a day through its plant at Alvarado for the public supply of the transbay cities. Its average aninual withdrawal during the five-year period 1904-1908, inclusive, was $1,541,000,000$ gallons, or 4,730 acre-feet, and during the five-year period 1909-1913, inclusive, $2,630,000,000$ gallons, or 8,075 acre-feet. Its withdrawal in the year. 1913 was $2,856,000,000$ gallons, or 8,770 acre-feet-an amount sufficient to lower the water table about $1 \frac{2}{3}$ feet a year if drawn entirely from the Alameda Creek supply.

\section{IRRIGATION.}

Water for irrigation in this area is obtained entirely from the underground reservoir. Data available are not sufficient to make a satisfactory estimate of the amount of ground water so used. The drought of 1911-12 and 1912-13 focused the attention of the whole community on irrigation, and it is safe to say that two-thirds of the irrigation wells in this region were sunk in the last three years. This development will continue to be rapid even in years of normal rainfall. Grain farming is rapidly being displaced by the raising of more profitable crops, such as alfalfa and tomatoes. The grain and grain-hay crops are almost never irrigated, but both alfalfa and tomatoes will be grown under irrigation.

1 The future water supply of San Francisoo from the conservation and use of its present resourcen, pp. 305-308, Oct. 31, 1912. 
The following estimate of the amount of water needed for the irrigation of the crops grown on the Niles cone and vicinity has been prepared from the work of Frank Adams and is based on the acreage of the different crops as determined for 1913:

Estimate of water needed for irrigating crops now grown on the Niles cone and vicinity.

[Based on the work of Frank Adams, U. S. Dept. Agr. Bull. 158, pt. 2, and Bull. 254.]

\begin{tabular}{|c|c|c|c|c|c|}
\hline \multirow{2}{*}{ Character of crop. } & \multicolumn{2}{|c|}{$\begin{array}{l}\text { Depth of water needed } \\
\text { in an average year } \\
\text { (inches). }\end{array}$} & \multirow{2}{*}{$\begin{array}{c}\text { Area } \\
\text { (acres). }\end{array}$} & \multicolumn{2}{|c|}{$\begin{array}{l}\text { Requirement } \\
\text { (acre-feet). }\end{array}$} \\
\hline & Maximum. & Minimum. & & Maximum. & Minimum. \\
\hline 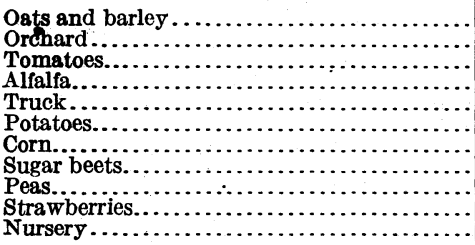 & $\begin{array}{l}12 \\
18 \\
30 \\
30 \\
30 \\
12 \\
12 \\
12 \\
30 \\
30 \\
12\end{array}$ & $\begin{array}{r}8 \\
12 \\
24 \\
18 \\
24 \\
8 \\
8 \\
8 \\
24 \\
24 \\
8\end{array}$ & $\begin{array}{r}5,170 \\
2,900 \\
1,350 \\
2,430 \\
1,300 \\
380 \\
340 \\
75 \\
25 \\
65 \\
550\end{array}$ & $\begin{array}{r}5,170 \\
4,350 \\
3,375 \\
6,075 \\
3,250 \\
380 \\
340 \\
75 \\
50 \\
162 \\
550\end{array}$ & $\begin{array}{r}3,446 \\
2,900 \\
2,700 \\
3,645 \\
2,600 \\
252 \\
226 \\
-\quad 50 \\
63 \\
130 \\
366\end{array}$ \\
\hline & & & 14,585 & 23,777 & 16,378 \\
\hline
\end{tabular}

\section{LOSS BY EVAPORATION ON THE SALT MARSH.}

Duryea ${ }^{1}$ found the average annual loss by evaporation from a free water surface in three pans on dry land near San Jose, Cal., to be 56.7 inches for a period of two years, 1904 and 1905, and the average loss from two pans floating in water to be 44.7 inches for the same period. These experiments were made in the Santa Clara Valley about 18 miles from Niles and at about the same elevation. The conditions under which the experiments were made are therefore similar to those on the Niles cone.

Lee ${ }^{2}$ found through his experiments in Owens Valley, Cal., that the evaporation from a deep tank set in the soil was 66.9 inches for the season 1910-11. He further found (Table 57) that the evaporation from a land surface covered with a good growth of salt grass where the depth to ground water was nearly 2 feet, conditions similar to those on the salt marsh adjacent to the Niles region, amounted to 42.67 inches for the season of 1910-11. The evaporation from the land covered with salt grass was therefore 63 per cent of that from the free water surface in the tank set in the soil.

By combining the results of Duryea with those of Lee it is possible to reach some estimate of the amount of ground water lost by evaporation on the salt marsh at the foot of the Niles cone and adjacent

\footnotetext{
1 Duryea, Edwin, jr., Eng. News, Feb. 29, 1912, p. 382.

2 Lee, C. H., An intensive study of the water recources of a part of Owens Valley, Cal.: U. S. Geol. Survey Water-Supply Paper 294, Table 51, p. 119, and Table 57, p. 122, 1912.
} 
area. This salt marsh borders San Francisco Bay and along the sloughs is wetted by the tides each day. The average evaporation from a free water surface on the marsh is therefore believed to be somewhat less than that shown by Duryea's land-pan records and somewhat more than that shown by his floating-pan records. The mean between these two is 50.7 inches a year, which is believed to represent approximately the evaporation that would occur if land pans were installed on the salt marsh. Lee's data show that the evaporation over the land covered with salt grass is 63 per cent of that from the land pan; and 63 per cent of 50.7 inches is 32 inches, or 23 feet, which is approximately the depth of water that evaporates from the salt marsh each year. The area of the salt marsh is about 9,600 acres, and this area covered to a depth of $22_{3}^{2}$ feet is equivalent to 25,600 acre-feet. A part of the water that evaporates is sea water which enters through the sloughs and seeps into the banks of the sloughs, but the general observations which were made lead to the conclusion that most of it is shallow ground water supplied from Niles cone and adjacent tracts without passing beneath the denser clay beds. From the data at hand it seems reasonable to assume that the average annual loss of ground water by evaporation from the area under consideration is between 10,000 and 25,000 acre-feet.

\section{SEEPAGE INTO THE BAY.}

An attempt was made to estimate the quantity of water lost through seepage into the bay by comparing the salinity of the bay water with that of sea water, but the data were found to be too meager to warrant drawing any conclusions. However, as the water beneath the bay is confined under a thick bed of dense clay, and the artesian pressure is slight, and as the water table has the very gentle slope shown by the water contours $(\mathrm{Pl}$. X), the loss by seepage into the bay from either deep or shallow sources is probably small.

\section{RELATION OF WITHDRAWALS TO CONTRIBUTIONS.}

The estimated contribution of about 59,000 acre-feet in the season of 1913-14 to the underground supply of the Niles cone and adjacent tract west of the fault is exclusive of the water pumped or otherwise withdrawn during the period of rising water. The withdrawals for irrigation and the loss by evaporation are almost entirely outside of this period, but a considerable part of the pumping by the People's Water Co. is done while the ground water is rising.

The rising-water season of 1913-14 began in the later part of December and closed near the end of April.- The total amount of water pumped by the People's Water Co.from May 1 to December 31, 1913, was 6,000 acre-feet. The average annual loss by evaporation on the 
salt marsh and seepage into the bay is probably between 10,000 and 25,000 acre-feet. The annual requirement for irrigation of all the crops now grown, as computed in the table, is between 16,000 and 24,000 acre-feet, the minimum requirement being approximately the quantity used for irrigation at the present time and the maximum the quantity that will probably be required in the near future. The total annual draft of ground water, exclusive of the withdrawals during the period of storage, would therefore be between 32,000 and 55,000 acre-feet if the pumpage by the People's Water Co. remained the same as in 1913 and all the crops now under cultivation were irrigated.

Interchange of ground water may take place between the Niles cone and the alluvial cones farther north, but it may be assumed that the supply of these cones is required for their own irrigation and is not available for pumping on the Niles cone. The annual storage would therefore be, in the most favorable years, between one and two times the annual requirement under the prescribed conditions, but in the least favorable years between one-tenth and one-twentieth of these requirements. The average annual contributions are probably nearer the maximum than the minimum, but they are no doubt considerably less than the contributions of 1913-14.

After making allowance for the uncertainties entering into several of the factors involved, it appears that the ground-water supply of the Niles cone and adjacent areas under consideration west of the fault is little if any greater than the amount required by present developments and is hardly adequate for the full irrigation of the area and the present diversions by the Spring Valley and People's water companies. If further diversions are necessary for the public supplies of San Francisco or the transbay cities irrigation developments will probably have to be arrested unless provision can be made for conserving and utilizing the large quantity of flood water which runs to waste into the bay through Alameda Creek.

The northern portion of the valley area east of the fault has a larger ground-water supply in proportion to its extent than the area west of the fault. Its supply is believed to be at least adequate for the irrigation of the arable land that it contains, but from the vicinity of The Lagoon southward the materials of the water-bearing beds are of such a character that, in general, they do not yield water rapidly enough to meet the demands of irrigation.

\section{INCURSION OF SEA WATER.}

Some of the inhabitants of the region fear that with a lowering of the ground-water level the sea water will be drawn into the underground reservoir and the salt marsh will encroach upon the land. So far as is known to the writer, however, there is no definite basis for this fear-indeed, there is evidence that encroachment of the salt 
water is not likely to occur. The water of the bay is believed to be effectively shut off from the ground water. According to Mr. J. B. Rogers, a driller, coarse gravel containing a strong flow of good water was struck at a depth of about 196 feet in the well of the Morgan Oyster Co. off Mowrys Landing, and there are other freshwater wells in the bay. One well on the shore of the bay at Ravenswood, which flows at high tide but not at low tide, yields water having only 27 parts per million of chlorine. As most of the wells in the bay do not overflow at present it appears that the salt is kept out by the impervious character of the materials underlying the bay rather than by the excess pressure of the fresh water. A nearly impervious bed of blue clay lies below the bay and extends over the salt marsh. Below the bay it has a maximum thickness of at least 120 feet, but it thins in the direction of the land. This bed will probably prevent the entrance of salt water, except perhaps very locally, even if the head of the ground water is lowered below sea level.

\section{SUMMARY OF CONCLUSIONS.}

1. The ground water in the vicinity of Niles originates in the rain that falls upon the drainage basins tributary to this part of the Santa Clara Valley and to a small extent in the rain that falls upon the valley itself.

2. The Niles-Irvington fault, which cuts off the reentrant of the valley between Niles and Irvington, has a profound effect on the surface drainage and on the circulation of the ground water. It is the cause of an escarpment, west of Tule Pond and The Lagoon, which intercepts and diverts southward all the mountain streams between Niles and Irvington except Alameda Creek. It has also produced an underground dam which prevents the passage of ground water from the east to the west side of the fault except in small amounts.

3. The effectiveness of the fault as an underground dam is proved by the great differences that exist in the ground-water levels on opposite sides of it and also by the great differences in the interval required for the water levels on opposite sides to respond to rainfall.

4. The area east of the fault receives a part of its ground water from Alameda Creek and a part from the smaller streams that discharge upon this area; an area west of the fault occupied by the Niles cone and a tract between this cone and a line extending approximately from Irvington through a point on the Southern Pacific Railroad 1 mile southeast of Mowry station receives practically its entire ground-water supply from Alameda Creek. - The area supplied by Alameda Creek west of the fault therefore covers somewhat more than 20,000 acres, exclusive of the salt marsh. 
5. The contributions to the ground-water supply in the season of 1913-14 were unusually large. According to observations at 125 wells the water table in the area supplied by Alameda Creek west of the fault rose 11.32 feet during the replenishing period of this season, and it is estimated that the total amount of ground water contributed to this area in the season of 1913-14 was about 59,000 acre-feet, exclusive of the amount withdrawn during the period of rising water.

6. The contributions to the ground-water supply in the season of 1912-13 were unusually small. Over a large part of the area supplied by Alameda Creek west of the fault the water table did not rise at all, and even in the most favored localities it generally rose less than 2 feet. The total amount of ground water contributed to this area in the season of 1912-13 is estimated at only 2,600 acre-feet, exclusive of the amount withdrawn during the period of rising water.

7. The annual contribution of ground water to the Niles cone and the adjacent area under consideration west of the fault is therefore believed to range between 2,600 and 59,000 acre-feet, or between somewhat wider limits, exclusive of the withdrawals during the replenishing period.

8. In the area supplied by Alameda Creek west of the fault ground water is withdrawn by pumping at the plant of the People's Water Co. for consumption in the transbay cities, by pumping at many small plants for local irrigation, and by unavoidable loss through evaporation on the lowlands bordering the bay and seepage into the bay.

9. The annual withdrawals for consumption in the transbay cities averaged 4,730 acre-feet in the 5-year period 1904-1908, inclusive, 8,075 acre-feet in the 5-year period 1909-1913, inclusive, and 8,770 acre-feet in 1913, of which about 6,000 acre-feet was withdrawn outside of the replenishing period.

10. The estimated annual requirement of ground water for irrigation of all crops now grown on the Niles cone and on the adjacent area supplied by Alameda Creek west of the fault is between 16,000 and 24,000 acre-feet, the smaller quantity being approximately that now used for irrigation and the larger quantity that which will be required in the future.

11. It is estimated that the total annual withdrawal of ground water from the Niles cone and the adjacent area supplied by Alameda Creek west of the fault, excluding the withdrawals during the replenishing period but including the unavoidable loss through evaporation and seepage, would be 32,000 to 55,000 acre-feet if the pumpage for the transbay cities remained the same as in 1913 and all present crops were irrigated.

12. About 18,000 acre-feet is annually withdrawn by the Spring Valley Water Co. from the Alameda drainage basin above Niles for 
consumption in San Francisco. If this water were not diverted a large part would ultimately reach the underground reservoir of the Niles cone. If the withdrawals for this purpose are increased the contributions to the ground-water supply of the Niles cone and adjacent tract will be further decreased.

13. The ground-water supply of the Niles cone and the adjacent area west of the fault is little if any greater than the amount required by present developments and is hardly adequate for the full irrigation of the area and the present-scale diversions by the Spring Valley and People's water companies. If further diversions are necessary for the public supplies of San Francisco or the transbay cities irrigation developments will probably have to be arrested unless provision can be made for conserving and utilizing the flood water which now runs to waste.

14. The north end of the valley area east of the fault has a larger ground-water supply in proportion to its extent than the area west of the fault. Its supply is believed to be at least adequate for the irrigation of the arable land that it contains, but the portion of this area from the vicinity of The Lagoon southward is underlain by material that in general yields water too slowly for irrigation.

15. There is little danger, even with heavy pumping, that the area under consideration will be seriously damaged by the encroachment of sea water. 
$\ddots$ 